\title{
100 JAHRE WEIMARER VERFASSUNG
}

\section{YEARS WEIMAR CONSTITUTION}

\author{
Christoph Gusy \\ Universität Bielefeld
}

\begin{abstract}
ZUSAMMENFASSUNG. I. DIE DYNAMISCHE VERFASSUNG: WAS WIR ÜBER DIE WRV WISSEN (KÖNNEN). II. EIN BLICK IN DIE WRV - EINE ZUKUNFTSOFFENE UND ZUKUNFTWEISENDE VERFASSUNG. III. DIE VERDRÄNGTE REVOLUTION - GEGEN DIE MONARCHIE, NICHT GEGEN DIE REPUBLIK. IV. DIE REPUBLIK: KEIN ERSATZKAISER, SONDERN VOLKSGEWÄHLTER REICHSPRÄSIDENT. V. PARLAMENTARISCHE REGIERUNG - SCHWACH ODER GESCHWÄCHT? VI. PLEBISZITÄRE REPUBLIK? VII. „DAS FREIESTE VOLK DER ERDE“ - DIE GRUNDRECHTE. VIII. NEUTRALITÄT BIS ZUM SELBSTMORD?- DAS NATIONALSOZIALISTISCHE REGIME ALS LETZTE REGIERUNG DER REPUBLIK? IX. RÜCKBLICK: VON WEIMAR ZU „WEIMAR“ UND WIEDER ZURÜCK. X. DIE WRV ALS MEILENSTEIN DER DEUTSCHEN UND EUROPÄISCHEN VERFASSUNGSGESCHICHTE.
\end{abstract}

Auszug: Vor 100 Jahren, am 14.8.1919, trat die Weimarer Verfassung in Kraft. Ein Blick in ihren Text mag überraschen. Allgemeines und gleiches Wahlrecht; Mitwirkung des Volkes an der Gesetzgebung und Partizipationsmöglichkeiten der Bürger; Anläufe zu einer Kontrolle der Staatsgewalt durch Gerichte einschließlich eines Verfassungsgerichts; Sozialpartnerschaft durch Mitbestimmung; wirtschaftliche und soziale Garantien bis hin zum Mindestlohn; Recht auf Bildung und Erziehung zu „staatsbürgerlicher Gesinnung“ und Völkerversöhnung; Gleichberechtigung von Frauen und Männern, Chancengleichheit für einzelne benachteiligte Gruppen und im Bildungswesen, vielleicht sogar Ansätze von Nachhaltigkeit. Alles dies war in der Weimarer Verfassung bereits angelegt

\begin{abstract}
August 14th 1919, the Weimar Constitution came into force. A look into its text may come as a surprise. A general and equal right to vote; Participation of the citizens in the legislation and other participation opportunities of the people; Attempts to control the State power by courts including a constitutional court; Social partnership through participation; economic and social Guarantees including rules on the minimum wage; Rights to education towards a "Civic attitude" and an international reconciliation; Equal rights of women and men, equal opportunities for individual disadvantaged Groups and in terms of education, maybe even approaches of Sustainability. All this was already included in the Weimar Constitution.
\end{abstract}

Schlüsselwörter: Weimar Constitution, National Assembly, Democracy, Republic, Fundamental Rights, End oft he Republik 
Keywords: Democracy-Republic-President of the Republic- Parliamentary Government-Defence oft the Constitution

\section{DIE DYNAMISCHE VERFASSUNG: WAS WIR ÜBER DIE WRV WISSEN (KÖN- NEN)}

Der Blick zurück auf die Republik und ihre Verfassung ist überlagert von Bildern, Zuschreibungen und Bewertungen, die teils von Zeitgenossen, teils von späteren (Rechts-)Historikern auf uns gekommen sind. Diese hatten ihre Bedeutung zu ihrer Zeit, als sie entworfen oder tradiert worden sind. Doch welche Bedeutung können sie für uns und unsere Anschauung der ersten republikanischen Verfassung für Deutschland erlangen? Die in der Gegenwart angemahnte hinreichende Komplexität von Fragestellungen und Antworten scheint die Einfachheit und Einlinigkeit jener Bilder zu überfordern. Unsere Suche führt uns zu vier Quellen:

(1) Den Verfassungsarbeiten der Nationalversammlung und in ihrem Verfassungsausschuss: Sie sind jüngst neu recherchiert und veröffentlicht worden. ${ }^{1}$ Was später das geltende Recht werden sollte, ist im Weimarer Verfassungslaboratorium $^{2}$ entworfen, abgewogen und in Auseinandersetzung mit konkurrierenden Ideen beschlossen worden. Wir kennen jetzt die Motive und Beratungen genauer und können besser mit der tradierten Formel von der „improvisierten Demokratie $^{\text {" umgehen. }}{ }^{3}$ Gewiss: Die Situation der Verfassunggebung war plötzlich und ungeplant eingetreten. Die Verfassungsberatungen waren es nicht. Sie waren wissenschaftlich und politisch vorbereitet, fanden interessierte und engagierte Politiker im Verfassungsausschuss. Dessen Debatten vereinigten die besten Traditionen deutscher Verfassungsstaatlichkeit, 4 nicht nur den Rückgriff auf die Paulskirche 1849,5 und in Einzelfällen ausländische Vorbilder. ${ }^{6}$

(2) Dem Verfassungstext: Er kondensierte die Beratungen und Ergebnisse des Verfassungslaboratoriums in der Sprache der Zeit. Sie sollten das Verfassungsrecht auf neue Grundlagen stellen, waren zugleich aber auch vielfach offen und ausgestaltungsfähig. Die demokratische Republik war mit dem Akt der Verfassunggebung nicht einfach fertig. Zahlreiche Staatszielbestimmungen, Gesetzgebungsaufträge und Gesetzesvorbehalte indizierten: Die WRV suchte einen eigenständigen Weg zwischen Stabilisierung des rechtlichen Status quo und Offenhaltung des politischen Prozesses für die Aufgaben der Zukunft. Die Brücke zwischen beiden lag in einem starken legitimationsorientierten Grundzug: Was das Volk durch die Weimarer Konstituante begründet hatte, sollte durch seine Vertreter in den Parlamenten auf- und ausgebaut werden. Dazu sollten nicht bloß rechtliche Besitzstände dem Zugriff der Staatsgewalt durch Abwehrrechte entzogen werden. Vielmehr zeichnete der Text Grundelemente einer Verfassungsstaat-

1 Jörg-Detlef Kühne, Die Entstehung der Weimarer Reichsverfassung, 2018.

2 Ausdruck nach Walter Pauly, Grundrechtslaboratorium Weimar, 2004, S. 25 ff.

3 Thomas Eschenburg, Die improvisierte Demokratie, 1963 (nach Hugo Preuß 1918).

4 Ausdruck nach Schefold, in: Heinrich (Hg.), Deutsche Juristen jüdischer Herkunft, 1992 , S. 429, 452 (zu Hugo Preuß).

5 Dazu unübertroffen Kühne, Die Reichsverfassung der Paulskirche, 2. A., 1998.

6 Zum Kontext Wiederin, in: Dreier/Waldhoff (Hg.), Das Wagnis der Demokratie, 2018, S. 45. 
lichkeit voraus, an der Deutschland und Europa erst viele Jahrzehnte später erneut angekommen sind. Dass deren Realisierung nicht allein durch Anordnungen in einer Verfassungsurkunde herbeibefohlen werden konnte, war den Abgeordneten in Weimar stets bewusst.

(3) Der Verfassungsauslegung der Staatsrechtswissenschaft: Sie war im Gegensatz zum späteren Grundgesetz weniger verfassungsgerichtlich dominiert. Zwar hatte die WRV mit dem Staatsgerichtshof erstmals ein eigenes Verfassungsgericht eingeführt. ${ }^{7}$ Damit griff sie der Verfassungsentwicklung im Vergleich zu anderen Staaten weit voraus. Doch waren ihm nur begrenzte Aufgaben zuerkannt, sein Ausbau durch Gesetz und Rechtsprechung noch in der Entwicklung. So blieb die Auslegungsaufgabe in hohem Maße der Rechtswissenschaft übertragen, die vor grundlegend neuen Aufgaben stand. Die Weimarer Verfassung basierte auf Grundideen (Republik, Demokratie), welche in der konstitutionellen deutschen Staatsrechtslehre negativ besetzt gewesen waren. ${ }^{8}$ Daher stellte sich für die Disziplin die Grundfrage: Konnte und sollte sie noch an ihre eigenen Traditionen und Prämissen anknüpfen, welche in der Vergangenheit eher in Distanz zu den neuen staatsrechtlichen Grundlagen gestanden hatten? Dies lief auf eine partielle Relativierung von Revolution und Republikgründung hinaus. ${ }^{9}$ Oder sollte die Disziplin nach der rechtlichen auch die wissenschaftliche Umwertung der neuen Errungenschaften nachvollziehen und sich so auf den Boden der neuen Verfassung stellen? Dies lief auf eine Neubegründung der eigenen theoretischen und methodischen Grundlagen hinaus. Die Staatsrechtswissenschaft stand so nicht nur vor der Verunsicherung durch einen neuen Gegenstand, sondern auch ihrer eigenen Grundlagen und Grundfragen. Zunächst würde die Disziplin mit sich selbst beschäftigt sein. Von ihr waren einigermaßen stabile und konsentierte Beiträge zur Auslegung der neuen Verfassung ungewiss und erst nach einer Neubestimmung ihrer Disziplin selbst zu erwarten.

(4) Der Verfassungspraxis: Der Text der WRV ist bis 1933 kaum geändert worden, er war also statisch. Die Staatspraxis war es nicht. Und damit auch nicht dasjenige, was sie z.T. mit intellektueller Vorarbeit, z.T. mit nachträglicher Billigung der Rechtswissenschaft aus der WRV gemacht hat. Es gab damals nicht bloß antidemokratisches Denken; auch war Weimar keine Republik ohne Republikaner und keine Demokratie oder Demokraten. Und dennoch ist festzuhalten: Der Blick auf die Verfassung der Republik zeigt ungeachtet ihrer kurzen Dauer eine dramatische Entwicklung der Verfassung von Text der WRV weg und aus ihr hinaus. Diese Dynamik und ihre Ergebnisse gilt es im Blick zu behalten, wenn von "der" Weimarer Verfassung geredet wird. „Die“ Verfassung der Republik war ein Prozess mit dramatischen Wandlungen weg von den Intentionen der Nationalversammlung und partiell auch weg vom Text. Was wir heute als "die“ Weimarer Verfassung kennen, war eher Prozess als Zustand. Und zentrale Bilder erscheinen aus der Retrospektive am ehesten als ein Momentaufnahme aus den Jahren 1926-1928. Als dynamische Verfassung war sie in weiten Bereichen dasjenige Recht, das in der Praxis und in der sich rasch entwickelnden Wissenschaft aus der WRV gemacht worden ist. Jener Wandel ist von Rechtsprechung und Rechts-

7 Dazu Horst Dreier, in: Thomas Simon (Hg.), Schutz der Verfassung, 2014, S. 317 ff, 151 ff, $161 \mathrm{ff}$.

8 Wiegand, Demokratie und Republik, 2017.

9 Darstellung bei Baldus, AöR 2002, 97. 
wissenschaft teils vorgedacht, teils mitgegangen, teils gerechtfertigt worden. Und so ist sie dann auch auf uns gekommen.

Aber nicht nur die Verfassung der Republik und ihrer Quellen befand sich in raschem Wandel. ${ }^{10}$ Auch ihre Rezeption durch Politik, Zeitzeugen und Zeitgeschichte nahmen nach 1933 und nach 1945 ihre eigene Entwicklung. ${ }^{11}$ Wichtige verfassungsloyale republikanische Traditionen brachen nach dem Ende der Republik ab und wurden erst später wieder entdeckt. Wichtige Kritiker der Republik konnten hingegen fortwirken und setzten später als Zeitzeugen dasjenige Bild von der WRV fort, an dem sie vor 1933 mitgewirkt hatten. Die junge Bundesrepublik hat die Weimarer Republik aus der Sicht ihrer Kritiker kennengelernt. Und sie hat sie auf der Suche nach „Lehren“ kennengelernt, welche für das Grundgesetz und seine Auslegung aus dem Schicksal der WRV gezogen werden könnten. Seit der Erkenntnis: „Bonn ist nicht Weimar“12 und „Berlin ist nicht Weimar" sind solche Perspektiven in den Hintergrund getreten. Diese Erkenntnis eröffnet neue Möglichkeiten, aber auch neue Herausforderungen. Auch mit wachsendem historischen Abstand bliebt die Suche nach der Verfassung jenseits solcher "Lehren“ und „Erfahrungen“ ein fortdauerndes Anliegen auf der Suche nach der Entwicklung von Verfassungsrecht und Verfassungsstaatlichkeit.

\section{EIN BLICK IN DIE WRV - EINE ZUKUNFTSOFFENE UND ZUKUNFTWEI- SENDE VERFASSUNG}

Wie modern die WRV damals war, zeigt ein Blick in die zeitgenössischen Verfassungen der deutschen Länder und anderer Staaten. ${ }^{13}$ Manches stand dort in der Sprache der Zeit, die nicht mehr in allen Teilen unsere Sprache ist. Und das allermeiste war nicht einfach vorgeschrieben, sondern aufgegeben. Darin lag kein Mangel, sondern weise Selbstbeschränkung: Eine moderne Sozialordnung kann nicht einfach herbeibefohlen und festgeschrieben werden, erst recht nicht in Zeiten allumfassenden Mangels wie 1919. Und sie muss stets fortgeschrieben und aktualisiert werden. Auch das war den Weimarer Verfassunggebern bewusst. Und wie komplex die Realisierung solcher Verfassungsaufträge ist, zeigen Anläufe zu ihrer Umsetzung bis in die Gegenwart. Die Weimarer Verfassung war eine gute, zukunftsoffene und zukunftweisende Verfassung in ihrer Zeit.

Dieser Befund kontrastiert vielen geläufigen Beschreibungen des Verfassungswerks. Oft fand sich eine tendenziell negative Einschätzung, und zwar aus zwei Richtungen. Die eine stellte auf das Verfahren der Nationalversammlung ab: Die Beratungen seien retrospektiv und stark auf die deutsche Vergangenheit konzentriert, ihr Werk insgesamt kompromisshaft gewesen. Das geht an der Eigenart demokratischer Verfassunggebung vorbei: Diese basiert fast stets auf breiten Mehrheiten und unterliegt daher hohen Konsens- und Kompromissanforde-

10 Jüngste Überblicke: di Fabio, Die Weimarer Verfassung, 2018; Gusy, 100 Jahre Weimarer Verfassung, 2018 (dort mit Nachw. zum gesamten Text dieser Abhandlung).

11 Grundlegend Ullrich, Der Weimar-Komplex, 2009. Zuvor Gusy (Hg.), Weimars lange Schatten, 2003.

12 F. Allemann, Bonn ist nicht Weimar, 1956.

13 Kailitz, Nach dem Großen Krieg, 2017. Zum aktuellen Forschungsstand Dreyer/Braune (Hg.), Weimar als Herausforderung, 2016; dies., Republikanischer Alltag, 2017. 
rungen. Dabei ist der Kompromiss kein Nachteil, sondern eher ein Vorteil. Er kann nicht schlecht sein, weil er ein Kompromiss ist, sondern nur, weil sein Inhalt schlecht ist. Hier setzte die zweite Kritikrichtung ein. In der WRV hätten unterschiedliche, z.T. widersprüchliche Elemente nebeneinander gestanden. ${ }^{14}$ Sie habe zu wenig Rücksicht auf die Not der Zeit genommen, zu viel versprochen und zu wenig geschaffen. Wahlsystem und plebiszitäre Elemente seien unzweckmäßig und unabgestimmt, das Verhältnis der gewählten Organe Reichspräsident und Reichstag sei in ein offenes Gegeneinander gemündet. Die Grundrechte seien leerlaufend, ihr Verhältnis zur Gesetzgebung unklar gewesen, die sozialen Verbürgungen nicht eingelöst worden. Aus einer solchen Sicht erscheint die Verfassung als Ganzes wie auch in ihren Teilen als eine Summe uneingelöster Versprechen. Die Quintessenz der Kritik lässt sich zusammenfassen: Genetisch sei sie das „Produkt einer gescheiterten Revolution“. ${ }^{15}$ Sie habe nicht integrierend gewirkt. Eine solche Kritik steht zum geschilderten Verfassungsinhalt in einem auffälligen Kontrast. Sie spiegelt eher hochfliegende Erwartungen einer Minderheit der Revolutionäre von 1918 und manche Befürchtungen mancher Bürger: Keine Räterepublik, keine Sozialisierung, selbst die staatliche Kontrolle über die Grundstoffindustrie nur als Gesetzgebungsauftrag (Art. 155 Abs. 4 WRV), dagegen die Grundrechte einschränkbar und durch Gesetze relativierbar. Im Licht solcher Kritik erscheint die WRV als falsche Verfassung zur falschen Zeit.

Solche Thesen waren geeignet, die früher verbreitete „Geburtsfehlertheorie“ der Republik zu stützen. ${ }^{16}$ Der Untergang der Republik war danach bereits in ihren Gründungsmängeln angelegt, und zu diesen Gründungsmängeln zähle insbesondere die Verfassung. Die „Fehlgeburt einer Republik“ begründete danach Geburtsfehler des staatlichen Neuanfangs nach 1918, der Republik und ihrer Verfassung: Kompromisshaftigkeit, Improvisation, Relativismus. Verstärkt wurden und werden derartige Einschätzungen durch das Scheitern der Republik, das im Lichte der Geburtsfehler eher als Konsequenz der Verfassung denn als deren Beseitigung durch äußere Einflüsse erscheinen musste. Dass eine „überforderte $\operatorname{Re}$ publik" auch eine überforderte Verfassung haben könne, ${ }^{17}$ geriet in einer solchen Perspektive leicht aus dem Blickfeld. Aber nicht nur das Ganze der Verfassung, sondern auch wesentliche ihrer Teile sind Objekte nachwirkender Bilder. Das vielleicht stärkste ist die Formel vom „Ersatzkaiser“, von der Republik als ErsatzMonarchie. Es spielte in der Weimarer Nationalversammlung keine Rolle und wurde vereinzelt von Kritikern der Verfassungspläne Preuß' benutzt. Die Formel entstammt ersichtlich nicht der Ebert-Zeit, dessen Amtsführung mit diesem Prädikat von niemandem gleichsam „geadelt“ wurde, sondern ist erkennbar von der späteren Hindenburg-Zeit geprägt. Ein anderes Bild ist dasjenige von der „Neutralität“ bzw. „Wehrlosigkeit“ der Republik, welche angeblich - „neutral bis zum

14 Darstellung und Antikritik bei Gusy, Journal der Juristischen Zeitgeschichte 2011, 47.

15 Nach Büttner, Die überforderte Republik, 2008, S. 120, das „überwiegende“ Urteil bei Zeitgenossen und in der Geschichtsschreibung. „Geburtsfehlertheorie“ z.B. bei Fraenkel, Deutschland und die westlichen Demokratien, 1994, S. 201. Mängeldiagnose bei Roellecke, Der Staat 1996, 599 (Nachw.). Überblick über die Ursachendiagnosen Schulze, in: Erdmann/Schulze, Weimar Selbstpreisgabe einer Staatsform?, 1984, S. 23, der über 100 belastende Faktoren anführt.

16 Zuletzt Lammert, in: Lehnert u.a. (Hg.). Hugo Preuß, Gesammelte Schriften III, 2015, S. VIII: Die WRV war „besser als die damaligen Verhältnisse und doch eben nicht gut genug für die Verhältnisse, die sie ordnen sollte.“

17 Dazu Gusy, Der Staat 2016, 291. 
Selbstmord“ - den Lemmingen gleich ihrem eigenen Untergang entgegenstrebte. Konstitutionalisierte Neutralität statt Streitbarkeit: Ein Umstand, welcher auch die Einschätzung nahelegen kann, die Verfassung sei eben nicht gut genug gewesen, um die Republik zu retten. Das Bild war zu allen Zeiten schief. Mit denselben Mitteln, mit denen Ebert 1923/24 die Republik gerettet hat, ist sie 1932/33 beseitigt worden. Und vergleichbare Urteile zu Neutralität und Wehrlosigkeit fanden sich zur Verfassung des Kaiserreichs von 1871 niemals, obwohl doch auch dieses nahezu geräuschlos unterging. Es steht zudem quer zu anderen Bildern von der Republik, welche diese jedenfalls in der Phase bis 1923 als kämpfende Republik beschrieben oder deren späteren Untergang auch auf einen Missbrauch der Notstandsbefugnisse der WRV zurückführte. War die Republik wehrlos oder allzu wehrhaft - vielleicht gar gegen sich selbst? Damit zusammen hängt die Formel von der "Legalität“ von Hitlers Kanzlerschaft. In einem solchen Licht mag dann die ganze Republik als bloße Vorgeschichte der Hitlerzeit oder umgekehrt das nationalsozialistische Regime als letzte „verfassungsgemäße“ Regierung der Republik erscheinen. Dafür spricht wenig, war doch die Legalitätsformel in der Republik nahezu ausschließlich eine Propagandaformel der aufstrebenden NSDAP auf ihrem Weg zur Macht, die damals mit ganz heterogenen Inhalten gefüllt wurde. ${ }^{18}$ Nach 1933 verschwand sie alsbald und wurde erst nach 1945 mit partiell alten, partiell neuen Inhalten wiederbelebt. Dass an Hitlers Kanzlerschaft mehr als der formale Ernennungsakt „legal“ war, ist längst in das Reich der Fabel verwiesen.

Die Bilder sind offenbar Ausdruck eines Distanzierungsbedürfnisses von der Republik und ihrer Grundordnung; eines Bedürfnisses, welches bereits aus der Republik stammt und sich später historisch verfestigt hat. Auch legte die lange Zeit hindurch verbreitete Sichtweise von der Republik als Phase der gelingenden Kultur und der misslingenden Politik nahe, die Verfassung als Recht des politischen Prozesses diesem Bereich und damit dem misslungenen Sektor zuzuweisen. Vielleicht finden sich deshalb auch keine positiven Verfassungsbilder. Inzwischen sind solche Bildern einer differenzierenden Betrachtung gewichen: Weder war die Kultur in allen Teilen gelungen noch die Politik in allen Teilen misslungen noch standen beide Bereiche einfach nebeneinander. ${ }^{19}$ Eine solche differenzierende Sicht gilt es auch auf die Verfassung anzuwenden.

\section{DIE VERDRÄNGTE REVOLUTION - GEGEN DIE MONARCHIE, NICHT GE- GEN DIE REPUBLIK}

Die Revolution von 1918 hatte sich gegen die Monarchie, die Diktatur der OHL und den permanenten Belagerungszustand gerichtet. Sie hatte sich nicht gegen die Republik gerichtet, sondern war ihr vorausgegangen. ${ }^{20}$ Die Arbeiter- und Soldatenräte waren schon da, als Parteien und die etablierte Politik noch nach Orientierung suchten. Das Bild von ihnen war lange Zeit hindurch primär durch den folgenden Bürgerkrieg und die in ihm verübten Übergriffe und Verbrechen

18 Dazu Rüffler, Vom Münchner Landfriedensbruch bis zum Mord von Potempa, 1994.

19 Büttner, Die überforderte Verfassung, 2008, S. 11 ff, 498 ff; Buchner, Um nationale und republikanische Identität, 2001, S. 7 ff, 346 ff. Zu den Verfassungsbildern Büttner, in: Dreyer (Hg.), Herausforderung (Anm. 1), S. 81.

20 Ausführlich Joachim Käppner, 1918 - Aufstand für die Freiheit, 2018 (Nachw.); Gusy, Recht und Politik 2018,135. Zur Vorgeschichte Lothar Machtan, Die Abdankung, 2. A., 2008. 
geprägt. ${ }^{21}$ Aus der Rückschau lässt sich festhalten: Es gab Räte, die gut funktioniert haben; solche, die schlecht funktionierten und solche, von denen keine nennenswerte Tätigkeit ausging. Die Ordnung dieses Systems war hochgradig informell, beruhte eher auf Absprachen und Programmen. Sie wurde jedoch mangels Alternativen - von der Rechtsprechung damals und später als Übergangsordnung anerkannt.

Aus der Retrospektive wirkt geradezu erstaunlich: Der Weg von der Revolution zur Weimarer Verfassung verlief auf der Ebene politischer und staatsrechtlich relevanter Entscheidungen planvoll und konsequent. Bereits drei Tage nach der Revolution wurden neue Grundrechte bürgerlicher, politischer und sozialer Art statuiert. ${ }^{22}$ Wenige Tage später begann H. Preuß im Auftrag der Volksbeauftragten mit der Ausarbeitung von Entwürfen für eine Verfassung. Und am 25.11.1918, zwei Wochen nach der Revolution, beschloss eine Reichskonferenz der Räte aus Reich und Ländern, die Verfassunggebung einer zu wählenden Nationalversammlung zu überantworten. Erst nachdem der Rat der Volksbeauftragten den Wahltermin diskutiert hatte und damit die Frage nach dem Ob von derjenigen über das Wann überlagert erschien, wurde das Ergebnis dem obersten Räteorgan, dem Reichskongress, vorgelegt. In ihm verfügte die (M)SPD über eine klare Mehrheit, während z.B. R. Luxemburg und K. Liebknecht kein Mandat erlangt hatten. Fünf Wochen nach der Revolution wurde hier die Entscheidung für die Nationalversammlung bestätigt (16.-19.12.1918), die einen Monat später gewählt wurde (19.1.1919), Anfang Februar zusammentrat und drei Monate nach der Revolution mit dem Gesetz über die vorläufige Reichsgewalt (10.2.1919) die demokratische Republik vorläufig etablierte. Das Gesetz enthielt wichtige Vorentscheidungen und Weichenstellungen für die Weimarer Verfassung. Praktisch allen diesen Entscheidungen lagen intensive und scharfe Diskussionen, menschliche und politische Zerwürfnisse, aber auch demokratische Wahlen und Entscheidungen mit klaren Mehrheiten zugrunde. Die Gegner der Nationalversammlung waren auch in den Räten in der Minderheit, und die Mehrheit der Wähler bestätigte überall den republikanischen Kurs. So rasch die Räte etabliert waren, so rasch verschwanden sie wieder. Die kurzlebigen Räterepubliken in München und Bremen verfügten über keine vergleichbare Legitimation. Sie konnten sich schon nach kürzester Zeit nur noch mit Gewalt halten und gegen ihre Niederwerfung mit Waffengewalt keine breite öffentliche Unterstützung mobilisieren. Aus der Retrospektive mögen Geschwindigkeit, Kontinuität und breite Legitimation des Weges zur demokratischen Republik erstaunen. Und sie lösen erst recht Erstaunen aus angesichts der Revolutions- und Rätelegenden und -historien, welche den Blick auf jene kurze Phase der deutschen Entwicklung verstellt haben. Der „Krieg, den keiner kennt“, der die Gründung der neuen Ordnung überschattete, war ein Kampf der Minderheiten gegen die Mehrheitsentscheidung für eine republikanische Ordnung von unterschiedlichen Seiten: Teils gegen die demokratische Revolution, teils für deren Fortsetzung. In jenem Bürgerkrieg traten bewaffnete Gewalt und eine dramatisierende Gewaltrhetorik nebeneinander und auseinander. Sie sollten die Gründung der Republik überschatten. Aber die Räte

21 Näher Platthaus, Der Krieg nach dem Krieg, 2018; Mark Jones, Am Anfang war Gewalt, 2017; Robert Gerwarth, Die Besiegten, 2017.

22 Aufruf v. 12.11.1918, RGB1. S. 1303. 
waren in Deutschland damals zu keinem Zeitpunkt eine mehrheitsfähige und damit demokratisch aussichtsreiche Alternative.

Die Nationalversammlung war am 19.1.1919 nach dem von den Volksbeauftragten eingeführten Verhältniswahlrecht gewählt worden. Die Herabsetzung des Wahlalters auf 20 Jahre und die Einführung des Frauenwahlrechts hatten keine Mehrheit der sozialistischen Parteien erbracht, sondern eine Dreiviertelmehrheit der Oppositionsparteien aus der Monarchie. In der kurzen Zeit zwischen Revolution und Wahltag war für eine Neuorganisation des Parteiwesens keine Zeit geblieben: Am Beginn der Republik standen das tradierte Parteienspektrum, z.T. unter neuen Namen, und aus der Monarchie bekannte Politiker. ${ }^{23}$ Die Friedensmehrheit des Jahres 1917 wurde zur Weimarer Koalition des Jahres 1919 und musste nun „die Suppe essen“ (Ludendorff), ${ }^{24}$ die ihr Monarchie und Militär eingebrockt hatten. Die Ratifikation des Vertrages von Versailles sollte zum Menetekel der Konstituante werden. In Plenum und Verfassungsausschuss herrschte eine ganz unrevolutionäre Stimmung. Ihre Arbeitsweise näherte sich rasch derjenigen des Reichstags an. Organisationsfragen, Geschäftsordnung, Anfragen, Debatten, Enqueten: Sie standen in der Tradition des deutschen Parlamentarismus.

Dass die Nationalversammlung nicht in Berlin tagte, war wohl auch Sicherheitsbedenken geschuldet, die sich als unbegründet erweisen sollten. Dass sie in Weimar tagte, war nach neueren Forschungen neben Verkehrs-, Versorgungsund Sicherheitsaspekten einer Kompromisssuche mit den süddeutschen Staaten geschuldet, die sich gegen Berlin als Verhandlungsort ausgesprochen hatten. ${ }^{25}$ Die Wahl des Ortes war also mehr und anderes als eine Flucht vor dem Volk. Der Vorsitzende des Verfassungsausschusses dankte der Stadt für ein friedliches Obdach, wo wir dieses Werk errichten und bauen konnten, und nannte das Ergebnis erstmals "Weimarer Verfassung“.26 Mit diesem Namen sollte sie fortan verknüpft sein, und dieser Name sollte später auch die von ihr verfasste Weimarer Republik bezeichnen.

\section{DIE REPUBLIK: KEIN ERSATZKAISER, SONDERN VOLKSGEWÄHLTER REICHSPRÄSIDENT}

Kaum ein Abschnitt der WRV ist mit derart zahlreichen Mythen umgeben wie derjenige über den Reichspräsidenten. Die wohl meist zitierte Umschreibung des Reichspräsidentenamtes spielte bei den Beratungen kaum eine Rolle und blieb dort eine selten verwendete Oppositionsformel gegen die Pläne von Preuß. Die Figur des „Ersatzkaisers“. ${ }^{27}$ Preuß sollte eine Schlüsselrolle bei den Vorarbei-

23 Zum Parteiensystem und seinem Wandel Dowe u.a., Parteien im Wandel, 1999.

24 Ritter/Miller, Die deutsche Revolution 1918/19, 2. A., 1975, S. 25 ff. Zum Versailler Vertrag in der Nationalversammlung Verhandlungen des Reichstags, Stenographische Berichte (zit. Verh. des RT), Bd. 327, S. 1082 ff; 1115 ff, 1136 (Abstimmungsergebnis), 1139 ff. Zum Vertrag und seinen Wirkungen Tooze, Sintflut, 2015, S. 335 ff. Zur „unrevolutionären“ Arbeit Abg. Cohn, Verh. des RT, Bd. 327, S. 1210.

25 Holste, Warum Weimar?, 2018.

26 Abg. Haußmann, Verh. des RT, Bd. 328, S. 2083. Einsetzung des Verfassungsausschusses ebd., Bd. 326, S. 502 .

27 Die Formel wurde eher kommentierend von Beobachtern aus dem liberalen Spektrum verwendet; Meinecke, Erinnerungen 1901-1919, 1949, S. 259; zurückhaltend Apelt, Geschichte der 
ten zur WRV einnehmen. ${ }^{28}$ Von den Volksbeauftragten eingesetzt stellten seine Vorentwürfe die Weichen für eine bürgerliche Verfassung. Sie erfuhren bereits vor ihrer Einbringung in die Nationalversammlung erhebliche Veränderungen durch Reichs- und Landesregierungen. Und in Weimar wurden die zur Vollverfassung ausgebaut und erneut erheblich verändert. Preuß hat diesen Prozess loyal erläuternd und kommentierend begleitet und sich das Ergebnis zu eigen gemacht. Er war der Initiator, aber die WRV hatte viele Väter (und einzelne Mütter).

Besonders umstritten blieb in den Verfassungsberatungen sein Konzept vom Reichspräsidenten als politischem "Gegengewicht". Hier vereinten sich tradierte Ideen des deutschen Liberalismus mit zeitgenössischen Thesen zum Parlamentarismus ${ }^{29}$ von der „parlamentarischen Regierung in ihrer wahren Form“. Die dort postulierte Notwendigkeit eines Gleichgewichts zwischen Exekutiven und Legislative beschrieb ein konstitutionelles, kein parlamentarisch-demokratisches Verfassungssystem. Katalysator der Rezeption jener schon damals veralteten Theorie war ein gewisses liberales Misstrauen gegen Parlamente, allgemeines und gleiches Wahlrecht. ${ }^{30}$ Nach Preuß sollte die Volkswahl dem Reichspräsidenten die Legitimation verleihen, parlamentarischen Fehlentwicklungen entgegenzuwirken. Die dazu notwendigen Kompetenzen sah er im Appell an das Volk durch Auflösung des Reichstags und Initiierung von Volksabstimmungen. Umgekehrt sollte sein parlamentarisches Gegengewicht durch Einleitung eines Abwahlverfahrens gegen den volksgewählten Präsidenten einzuleiten berechtigt sein (Art. 43 Abs. 2 WRV).

Solche Pläne stießen in der Nationalversammlung auf ein geteiltes Echo. Die bürgerlichen Koalitionsparteien äußerten sich tendenziell zustimmend. Die Opposition von rechts stellten eher Vergleiche zur vergangenen Monarchie an. Hingegen betonten SPD-Abgeordnete das Grundprinzip der "parlamentarischdemokratischen Organisation des Reichs“. ${ }^{11}$ Sie kritisierten eine im Vergleich zum früheren Kaiser sowie zum französischen und amerikanischen Präsidenten „höhere uneingeschränktere Macht“ des Staatsoberhaupts. Hinsichtlich der Wahl wurde kein Bedürfnis gesehen, vom Verfahren der Nationalversammlung abzuweichen, die Ebert zum Vorläufigen Reichspräsidenten gewählt hatte: Und bei der

WRV, 2. A., 1964, S. 201. Zu Ebert Mühlhausen, Friedrich Ebert, 2. A., 2007, S. 775 ff. Zu Hindenburg Pyta, Hindenburg, 2007. Jüngst Kielmannsegg, in: Dreier/Waldhoff aaO., S. 219.

28 Hugo Preuß, Gesammelte Schriften, 5 Bände, 2007 ff; zu den Verfassungsarbeiten Lehnert/Müller/Schefold (Hg.), Hugo Preuß, Gesammelte Schriften III, 2015. Biografisch Michael Dreyer, Hugo Preuß, 2018 (Nachw.).

29 Redslob, Die parlamentarische Regierung, 1918. Rezeption bei Preuß, Verh. des RT 326 , S. 291. Zu seiner Redslob- Rezeption Schönberger, Das Parlament im Anstaltsstaat, 1997, S. 293, 384 ff, 399 f. Vergleichbare Anklänge damals auch bei Max Weber, Gerhard Anschütz und Friedrich Meinecke.

30 Dazu Llanque, Demokratisches Denken im Krieg, 1997, S. 179 ff, 252 ff u. pass.; ders., in Gusy (Hg.), Demokratisches Denken in der Weimarer Republik, 2000, S. 38.

31 Preuß, Verh. des RT, Bd. 328, S. 2073; Abg. Katzenstein ebd., S. 2075. Zustimmend zu Preuß Abg. Koch ebd., S. 393 („in ernster Stunde ein Gegengewicht gegen die Parlamentsroutine“); Abg. Spahn ebd., S. 383; Bd. 328, S. 2078; zum Unterschied von Demokratie und Parlamentarismus Abg. Ablaß, Verh. des RT. Bd. 336, S. 232. Dagegen von rechts Abg. v. Delbrück, Verh. des RT Bd. 326, S. 383 f; Abg. Düringer ebd., Bd. 328, S. 2089 („Dekorationsstück“, „schwach“); Abg. Heinze ebd., S. 399 f; Bd. 328, S. 2095; für die SPD Abg. Fischer, Verh. des RT, Bd. 326, S. 374 (Zitate). Zur Entstehungsgeschichte Mühlhausen (Anm. 15), S. 192 ff. 
Ausgestaltung des Präsidentenamtes wollten ihre Redner keine Rücksicht darauf nehmen, dass mit Ebert ein Sozialdemokrat Inhaber des höchsten Amtes sei. Für sie standen institutionelle vor personellen Erwägungen, insbesondere Befürchtungen vor zukünftigen Amtsinhabern aus einer möglichen „reaktionären staatsstreichlüsternen Partei.“ Noch in der abschließenden Lesung kritisierte der SPDRedner die Volkswahl als "Möglichkeit einer bonapartistischen Plebiszitpolitik“.32 Die Befürchtung, dass das intendierte Gleichgewicht in ein Übergewicht des Präsidenten umschlagen könne, wurde schon damals geäußert. Für die USPD war der Reichspräsident überhaupt „unnötig“. Sie erwog ein mehrköpfiges Direktorium als Reichsspitze. ${ }^{33}$ Hinter jenen grundsätzlichen Erwägungen traten Erörterungen der Ausgestaltung des Amts des Staatsoberhaupts zurück.

In der Weimarer Verfassung wurde der Reichstag als erstes, das Staatsoberhaupt erst danach als zweites Organ der Republik erwähnt. Und das Parlament wurde gegenüber dem Staatsoberhaupt in mehrfacher Hinsicht verselbständigt und gestärkt. Die Nationalversammlung folgte Preuß eher im Hinblick auf konkrete Einzelnormen als auf die zugrunde liegenden Gleichgewichtsvorstellungen. Und zu deren Auslegung wurde in der Republik kaum je auf die Gleichgewichtsideen Bezug genommen. ${ }^{34}$

In parlamentarisch regierten Staaten verkörpert das Staatsoberhaupt eher als Nicht-Normierte und Nicht-Normierbare im Staat. Von daher kann es nicht überraschen: Jene grundsätzlichen Kontroversen über Amt und Funktion des Reichspräsidenten spiegelten sich in den konkreten Bestimmungen über seine Rechtsstellung kaum wieder. Die Einzelbestimmungen gingen von zwei Grundgedanken aus. Das Staatsoberhaupt war erst nach dem Reichstag geregelt und stand im Kontext nicht des Parlaments, sondern der Regierung. Die Volkswahl des Präsidenten (Art. 41 WRV) war nur erwähnt, aber nicht näher ausgestaltet. So war die Nationalversammlung der Anregung nicht gefolgt, die Wählbarkeit an die Geburt als Deutscher zu knüpfen. ${ }^{35}$ Das zweistufige Wahlverfahren mit einer Stichwahl wurde durch Gesetz eingeführt. ${ }^{36}$ Die lange Amtsdauer von 7 Jahren war als Basis von Stabilität und Kontinuität an der Staatspitze konzipiert.

Die Kompetenzen des Reichspräsidenten entsprachen dem in parlamentarischen Systemen Üblichen: Völkerrechtliche Vertretung des Reichs, Beamtenernennung und Begnadigungsrecht; Oberbefehl über die „Wehrmacht“ (Art. 47 WRV). ${ }^{37}$ Er konnte den Reichstag auflösen (Art. 25 WRV) und im Gesetzgebungs-

32 Abg. Katzenstein, Verh. des RT, Bd. 329, S. 2076. Gegensätzlich Preuß, Verh. des RT, Bd. 336, S. 277.

33 Abg. Cohn ebd., S. 404.

34 Nicht erwähnt bei Pohl, in: Anschütz/Thoma (Hg.), Handbuch des Deutschen Staatsrecht (zit: HDStR) I, 1930, S. 482 f; knapp genannt bei Anschütz, Die Verfassung des Deutschen Reichs, 14. A., 1933 (zit: WRV), S. 242 („Gegengewicht, welches zugleich die Selbständigkeit der vollziehenden im Verhältnis zur gesetzgebenden Gewalt verbürgt“).

35 Ursächlich war der Hinweis auf ehemals Deutsche, welche infolge der Gebietsveränderungen durch den Versailler Vertrag ihre Staatsangehörigkeit verloren. Abg. Philipp, Verh. des RT, Bd. 327, S. 1306; dagegen Abg. Haas ebd.

36 ReichsG v. 4.5.1920, RGB1 S. 849, m. Änd. Zum Wahlverfahren Pohl (Anm. 21), S. 467.

37 Hier sollte sich die in $\S 8$ Abs. 2 WehrG vorgesehene Zuständigkeitsverteilung zwischen Präsident und Wehrminister in der Republik verschieben: Während Ebert seine Kompetenzen auf den 
verfahren gegen ein vom Parlament beschlossenes Gesetz Volkabstimmungen einleiten (Art. 73 ff WRV). Von ersterer Kompetenz machte er regelmäßig, von letzterer nie Gebrauch. Umgekehrt unterlag er Kontrollrechten des Reichstags. Von Zeitgenossen wurden parlamentarische Misstrauensvoten gegen Regierungen (Art. $54 \mathrm{WRV}$ ) und die Gegenzeichnungspflicht präsidialer Maßnahmen durch die Regierung (Art. $50 \mathrm{WRV}$ ) hervorgehoben. Das Staatsoberhaupt sei „an die Regierung gekettet".38 Auffällig ist: Soweit das Gegengewichtskonzept konkret wurde, war es eher ein solches im Negativen durch Auflösungs-, Aufhebungs- oder Abwahlrechte, ein Gleichgewicht der Schwäche als der Stärke. ${ }^{39}$ Deren praktische Bedeutung sollte sich in der Folgezeit von den Prognosen der Verfassunggeber weit entfernen.

Das Konzept des Verfassungsausschusses zur Parlamentarisierung der Staatsgewalt einschließlich des Reichspräsidenten war eindeutig und wurde daher kaum expliziert. Ausgangspunkt war die Parlamentarisierung der Regierung durch die Notwendigkeit des Vertrauens der Abgeordnetenmehrheit und die Rücktrittspflicht im Misstrauensfall. Da die Regierung alle Maßnahmen des Staatsoberhaupts gegenzuzeichnen hatte (Art. $50 \mathrm{WRV}$ ), sei auf diese Weise auch der Reichspräsident an den Parlamentswillen gebunden. Das galt namentlich auch für die Reichstagsauflösung (Art. 25 WRV), die nur mit Zustimmung der Regierung zulässig sei, welche „ihre“ Wähler im Parlament vor Willkür schützen könne. Und wenn der Reichspräsident ausnahmsweise besondere Rechte im Wirkungsbereich des Reichstags ausübte, sollten dem besondere Parlamentsrechte gegenüberstehen: Das Misstrauensvotum in Falle parlamentarisch unerwünschter Regierungen (Art. 54 WRV) und das Aufhebungsverlangen gegenüber Notverordnungen (Art. 48 Abs. 3 S. 2 WRV). Die Frage, wie diese Kontrollrechte umgangen bzw. leerlaufen konnten, zählte zu den meistdiskutierten rechtswissenschaftlichen Fragestellungen zum Staatsorganisationsrecht. 40

Für die Staatspraxis der Republik folgenreich sein sollten drei Rechte des Staatsoberhaupts: Das Ernennungsrecht hinsichtlich des Reichskanzlers (Art. 53 WRV), das Auflösungsrecht gegenüber dem Reichstag (Art. 25 WRV) und die Notstandskompetenzen (Art. 48 WRV). Dass letztere eine zentrale Bedeutung erlangen sollten, hing wesentlich damit zusammen, dass während der Hälfte der Republik in wesentlichen Teilen der Republik ein Notstand herrschte. So wurde das Ausnahmerecht zu einem zweiten Normalfall, einer "Reserveverfassung“, welche die WRV immer mehr verdrängte und am Ende nahezu ersetzen sollte. ${ }^{41}$ Sein Anwendungsbereich wurde von polizeirechtsähnlichen Notständen hin zu Stö-

Minister delegierte, fanden sich unter Hindenburg gegenläufige Tendenzen einer Entgouvernementalisierung und Entparlamentarisierung der Befehlsgewalt.

38 Koellreutter, Das parlamentarische System, 1921, S. 6; ebenso Pohl, Die Auflösung des Reichstags, 1921.

39 Möller, in: Funke u.a. (Hg.), Festschrift für Bracher, 1987, S. 140. Aus der Sicht der Kritiker kombinierte die WRV zwei unvereinbare Modelle: Den volksgewählten Präsidenten der USA, der die Regierung bestimmte, aber auf den Kongress keinen Einfluss hatte; und den (damals) parlamentsgewählten Präsidenten Frankreichs, der die Kammer einberufen und auflösen konnte. Dabei entging den Zeitgenossen in Deutschland, dass der starken Exekutive in den USA ein starkes Gegengewicht durch eine von ihr unabhängige Volksvertretung gegenüberstand.

40 Näher dazu Gusy, 100 Jahre Weimarer Verfassung, 2018, S. 192 ff.

41 Dargestellt bei Gusy, Weimar - Die wehrlose Republik?, 1991. 
rungen im politischen Gefüge des Staates ausgeweitet, sein Instrumentarium erweitert und die Kontrollrechte abgebaut. Sie sollten je für sich und im Zusammenwirken Grundlage eines fundamentalen Verfassungswandels werden. Als Hauptproblem erwies sich nicht das befürchtete Übergewicht der Volksvertretung, sondern das schon in Weimar beschworene Übergewicht des Staatsoberhaupts. Statt der parlamentarischen Kontrolle der Regierung stand am Ende die präsidiale Kontrolle über den Reichstag.

\section{PARLAMENTARISCHE REGIERUNG - SCHWACH ODER GESCHWÄCHT?}

a) Das Konzept der Nationalversammlung: Wir haben gesehen: Die Nationalversammlung hatte die Republik als parlamentarische konzipiert. ${ }^{42}$ Deren Grundlage war das damals alternativlose Verhältniswahlrecht, das sich gegen als undemokratisch empfundene Praktiken von Mehrheitswahlen in der Monarchie richtete. Zwar machte die WRV nur die „Grundsätze der Verhältniswahl“ verbindlich und blieb so hinter den Volksbeauftragten zurück. Ursächlich waren Zweifel an der Vereinbarkeit von Verhältniswahl und parlamentarischem Regieren im Verfassungsausschuss. ${ }^{43}$ Doch war es namentlich die Rechtsprechung der StGHe, welche bis 1930 das Wahlsystem strikt formal verstand und so Versuchen, den Einzug von Klein- und Kleinstparteien in die Parlamente und deren Zersplitterung zu verhindern, entgegentrat. ${ }^{44}$

Hatten bis 1918 Politiker, Abgeordnete und Parteien in Reichs- und Landtagen ihr politisches Betätigungsfeld gefunden, so hatte der Bereich der Regierung dessen nahezu unübersteigbare Grenze markiert. Deren Überwindung war zentraler Gegenstand der Verfassungsreformdiskussionen im Weltkrieg gewesen. Schon das Gesetz über die vorläufige Reichsgewalt hatte die Regierungsform der Monarchie durch eine kollegiale Regierung abgelöst. Dieser Vorentscheidung folgte die Nationalversammlung (Art. 52 ff WRV). ${ }^{45}$ Aus der Sicht der Parlamentarismustheoretiker stand fest: „Die unzweifelhafte Absicht der Mehrheit der Weimarer Nationalversammlung war, ein sog. parlamentarisches Regierungssystem vorzuschreiben, das mindestens für die Regel und für die Dauer das Staatsoberhaupt von der Regierung abdrängt und diese einem von der jeweiligen Mehrheit des Parlaments gestützten Ministerium anvertraut“. 46 Das „parlamentarische System“ sollte Volksvertretungen und Regierung umfassen und diese auf den Willen des Volkes zurückführen. Der Verfassungsausschuss stand unter dem Eindruck der Vorentscheidungen aus dem Oktober. Bezeichnend war: Die genannten Zweifel an der Vereinbarkeit von Verhältniswahlsystem und Parlamentsregierung und von parlamentarischer und Präsidialgewalt waren im Kontext des Wahlrechts laut geworden und galten durch dessen Modifikation als beigelegt. Zur Frage der $R e-$ gierungsbildung wurde gleichfalls der Text der soeben verabschiedeten Oktoberreformen übernommen. Ein Wille, durch explizit angeordnete Kanzler- oder Ministerwahl die Parlamentsrechte noch stärker zu akzentuieren, fand sich in den

42 S. o. 4. Zum Demokratiekonzept der WRV Lübbe-Wolff, in: Dreier/Waldhoff aaO., S. 111.

43 Naumann, Verh. des RT, Bd. 336, S. 242.

44 Näher Gusy, Die Weimarer Reichsverfassung (zit: WRV), 1997, S. 115 ff; ders., 100 Jahre Weimarer Verfassung, 2018, 143 ff (Nachw.).

45 Verh. des RT, Bd. 336, 296 ff. v. Delbrück ebd., S. 296 ff; dagegen Preuß ebd., 300.

46 Thoma, HDStR I, S. 503. 
Beratungen kaum und im Verfassungstext gar nicht. Debatten kreisten um das Vorschlagsrecht des Kanzlers für die Minister (Art. 53 WRV), das Verhältnis von Kanzler- und Ressortprinzip (Art. 56 WRV) und die Ministeranklage (Art. 59 WRV); also Fragen, welche schon in der Monarchie Probleme aufgeworfen hatten. ${ }^{47}$ Generell folgte die Mehrheit Preuß, nach dessen Auffassung sich das Verhältnis von Kanzler und Ministern einer Regelung durch „spröde und unbiegsame Formen" des Rechts eher entziehe. Bei Koalitions- und Oppositionsparteien herrschte am Ende ein unterschiedlich bewerteter Konsens: Die neue Verfassung habe ein parlamentarisches Regierungssystem geschaffen.

Jener Plan geriet während der Verfassungsberatungen in seine erste Bewährungsprobe. In den 16 Monaten bis zur Auflösung der Nationalversammlung amtierten drei Kabinette unter drei verschiedenen Reichskanzlern. Infolge der eindeutigen Mehrheitsverhältnisse verliefen Koalitionsverhandlungen und Regierungsbildung im Zusammenwirken mit Reichspräsident Ebert vergleichsweise reibungslos. Die Reichstagswahlen des Jahres 1920 erschwerten die Kabinettsfindung. Die parlamentarische Regierungsbildung geriet in ihre erste Krise. Eine im Vergleich $\mathrm{zu}$ damaligen Landtagen unterdurchschnittlich ausgeprägte Bereitschaft der Fraktionen zur Zusammenarbeit, das Fehlen einer starken, nach unterschiedlichen Richtungen offenen politischen Mitte, Gegensätze zwischen Koalitionsparteien, eine um sich greifende Neigung von Kabinetten, bei Ablehnung einzelner Gesetzesvorhaben im Reichstag auch dann zu demissionieren, wenn dieser kein Misstrauensvotum ausgesprochen hatte, aus alledem resultierende kurze Amtsdauern der 20 Kabinette: Zeitgenössische Kritiker zeichneten das Bild, wonach die Republik die Regierungsgewalt weniger parlamentarisiert als vielmehr abgeschafft habe. Vor diesem Hintergrund sind gouvernementale und administrative Leistungen der jungen Republik von der Finanzreform (1919) über die Konversion von der Kriegs- zur Friedenswirtschaft, die Bewältigung der Hyperinflation und die Neuordnung der Arbeits- und Sozialfürsorge Zeugnisse einer hoch ausgebildeten Handlungsfähigkeit der Reichsexekutiven. ${ }^{48}$ Und ein Blick auf die nur wenige Häuser entfernt residierende preußische Staatsregierung zeigte: Parlamentarische Regierungsbildung sowie stabiles und durchsetzungsfähiges Regieren waren auch in der Republik nicht unvereinbar.

b) Die Reichsregierung zwischen staatsrechtlichem Anspruch und staatlicher Wirklichkeit: In der zeitgenössischen Staatsrechtslehre nahm die Regierung keinen prominenten Raum ein. Dass die Verfassungswirklichkeit Defizite gegenüber den Intentionen der WRV aufwies, war unumstritten. Anhänger der Republik sahen die Defizite weniger in der Verfassung als in ihren Vor- und Rahmenbedingungen wie Parteienvielfalt, Wahlergebnissen und externen Hindernissen wie Parlamentsauflösungen. Sie hoben die Chancen des neuen Regierungssystems hervor: Parlamentarisches Regieren sichere, dass Politik und Gesetzgebung nicht in Gegensatz geraten könnten zu dem, was der Mehrheit im Volk wünschenswert

47 Gegen starre Regelungen in der Verfassung Anschütz, DJZ 1917, 696, 698; Heller, (1931), in: Drath u.a. (Hg.), Gesammelte Schriften (zit.: GS), 2. A., 1992, Band II, S. 463, 468 f, 609. Eher retrospektiv zum Recht des Kabinetts Poetzsch-Heffter, HDStR I, S. 511; v. Bieberstein, HDStR ebd., S. 520 .

48 Historisch Mäding, in: Jeserich u.a., Deutsche Verwaltungsgeschichte IV, 1985, S. 92 ff; Berg ebd., S. 168 ff; 218 ff; Hettlage ebd., S. 177 ff. 
oder tragbar erscheine. ${ }^{49}$ Die Probleme wurden nicht primär auf die neue Staatsform, Verfassung oder Verhältniswahlrecht zurückgeführt. Bis zur Konsolidierung der politischen Verhältnisse wurden Hilfskonstruktionen vorgeschlagen, etwa besondere formelle Anforderungen an parlamentarische Misstrauensvoten oder die Beauftragung einer zurückgetretenen Reichsregierung als geschäftsführendes Kabinett für den Zeitraum bis zur Ernennung eines neuen, mehrheitsfähigen Kanzlers. In der praktischen Regierungstätigkeit könne bei fehlenden oder gar negativen Mehrheiten in den Volksvertretungen kommissarisch mithilfe der Notstandskompetenzen agiert werden.

In der öffentlichen Wahrnehmung setzt sich eher die Gegenauffassung durch. Verlustdiagnosen gegenüber dem Regierungssystem Bismarcks übergingen dessen Niedergang in der Monarchie und erst recht im Weltkrieg. Seit 1919 sei an die Stelle der monokratischen, allein auf die Person des Kanzlers zugeschnittenen Reichsregierung keine neu legitimierte Führung, sondern ein politisches Vakuum getreten. Das Vorschlagsrecht des Reichskanzlers für die Besetzung der Ministerposten (Art. 53 WRV) laufe infolge von Koalitionsabsprachen leer: Nicht der Kanzler, sondern die Koalitionsarithmetik bestimme die Ämterverteilung. Seine Richtlinienkompetenz (Art. 56 WRV) finde ihre Grenzen in der Bereitschaft der Minister koalierender Parteien, Vorgaben zu akzeptieren. So werde die Regierungsgewalt teils disfunktionalisiert, teils paralysiert. Verfassungsrechtlicher Anspruch und staatliche Wirklichkeit fielen auseinander. In den 20er Jahren steigerte sich die Diskussion zu einer - in späterer Terminologie - Diskussion über die Regierbarkeit der Republik. ${ }^{50}$ Die Regierung wirke wie ein Ausschuss des Parlaments, ${ }^{51}$ also ein schlecht funktionierender Ausschuss eines schlecht funktionierenden Parlaments. Damit gerate die WRV in einen Selbstwiderspruch: Einerseits setze sie eine handlungsfähige Reichsregierung voraus. Andererseits machten ihre organisatorischen Regelungen deren Bildung bzw. Stabilisierung unmöglich. Als Ursache wurde das in der WRV angelegte parlamentarische System ausgemacht. ${ }^{52}$ Solche Bilder waren gewiss einseitig und maßen die Realität an irrealen Ansprüchen. Dass eine „überforderte Republik“ eine überforderte Regierung haben würde, lag nahe. Auch wenn das vielfach tradierte Bild der Regierungsgewalt im Spagat zwischen gegenläufiger parlamentarischer und präsidialer Legitimation eher eine Momentaufnahme der Situation um 1926 darstellt: Die Instabilität der wechselnden Reichsregierungen zwischen 1920 und 1928 verlieh der Kritik vordergründig eine gewisse Berechtigung. Insbesondere vermittelten Regierungswechsel einen publizistischen Aufmerksamkeitsschub, demgegenüber die Stabilität etwa der preußischen Regierung eher unspektakulär blieb. Langfristig krisenverstärkend wirkte die Praxis der Reichspräsidenten, als Legitimationsreserve in die Blindstellen des parlamentarischen Systems einzurücken. Was un-

49 Thoma, HDStR I, S. 504. Zur Auflösungspraxis ders. ebd., S. 505 f. Zur Geschäftsregierung Thoma, HDStR I, S. 506 f; Anschütz, WRV (Anm. 21), S. 323 ff; zur Staatspraxis Gusy, WRV, S. 229. Zu den Notkompetenzen Anschütz ebd., S. 277 f. Prüfstein wurde das Budgetrecht des Parlaments; dazu Thoma, Zeitschrift für Öffentliches Recht 1931, 28; Poetzsch-Heffter, RuPVB1 1932, 321; Hensel, DJZ 1931, 1058; anders etwa Grau, HDStR II , 1932, S. 285; Löwenstein, AöR $1931 / 32,138$.

50 Poetzsch-Heffter, HDStR I, S. 519, forderte eine „einheitliche und kräftige Führung der Staatsgeschäfte“. Zur Verlagerung der Führung auf das Staatsoberhaupt ebd., S. 514.

51 Schmitt, Verfassungslehre, 1928, S. 265 , 266 f;

52 S. etwa Smend (1928), in: Staatsrechtliche Abhandlungen, 3. A., 1992, S. 119, $211 \mathrm{f}$. 
ter Ebert eher als Behelf für den Notfall erschien, wurde unter Hindenburg zunächst zur Normalität und dann zur antiparlamentarischen Strategie. ${ }^{3}$ Am Anfang stand die Ernennung eines Kanzlers, dem zuvor das Vertrauen entzogen war (Marx IV). Es folgte die präsidiale Suche nach Kanzlerkandidaten hinter dem Rücken und ohne Mitwirkung der Parteien (spätestens seit Brüning I). Die finale Krise entstand aus den „Kampfregierungen“ des Jahres 1932 gegen den expliziten Willen der Volksvertretung bei gleichzeitiger Drohung mit ihrer Auflösung (Art. 25 WRV), welche die Kontrollrechte des Reichstag leerlaufen ließ. Der Übergang von der parlamentarischen kontrollierten Präsidialgewalt hin zur präsidialen Kontrolle über das Parlament verfehlte die intendierte Wirkung: Eine Stabilisierung der Regierung gelangt nicht, stattdessen wurden das Parlament destabilisiert und die personellen Alternativen erschöpft. Und die verfassungsrechtlich unnötigen Parlamentsauflösungen der Jahre 1930 und 1932, welche eine Kaskade von Neuwahlen auf dem Höhepunkt der Wirtschaftskrise notwendig machten, lieferte das Parlament den Gegnern der Republik von rechts und links aus. ${ }^{54}$

Die staatsrechtliche Rechtfertigung jener Praxis musste eine bereits erwähnte größere Zahl von Sicherungen parlamentarischen Regierens in der WRV umgehen bzw. außer Kraft setzen. Dies war die Quintessenz der in Einzelheiten variierenden Lehre von den „Kampfregierungen“, 55 welche seit den 20er Jahren die Praxis Hindenburgs teils vordachten, teils legitimierten. Wir wissen inzwischen: Das war nicht nur praeter constitutionem, sondern in mancher Hinsicht contra constitutionem. Sie basierten auf zwei Grundgedanken: Der Gleichsetzung der Handlungsfähigkeit des Staates mit Handlungsfähigkeit der Exekutive, welche der WRV nicht entnommen, sondern entgegengesetzt wurde. Hier erschien der Reichspräsident als Faktor von Stabilität und Aktionsfähigkeit im Gegensatz zum Reichstag als Ort der Zersplitterung und Paralyse. Solche Ideen basierten auf der Entgegensetzung von geistig-politischem Staat und rechtlich angeordneter Staatsform. Und weil die Existenz des Staates seiner Form vorgeordnet wurde, musste letztere zurücktreten, wenn es um den Staat als Ganzes und als solchen ging. Positiv-rechtlicher Anknüpfungspunkt wurde der Art. 25 WRV, welcher die Auflösung des Reichstags zugleich ermöglichen und begrenzen sollte. Doch waren dort die Grenzen - wie schon in der Nationalversammlung gesehen, aber mangels besserer Formulierungsalternativen hingenommen - ganz unzulänglich formuliert. Sie wurden in der Folgezeit nicht operationalisiert, sondern zerredet. Die Folge war eine unbegrenztes Recht der Auflösung und (wichtiger noch) der Auflösungsdrohung, welche auch eingesetzt wurde, wenn als Folge der fälligen Neuwahlen Gewinne der radikalen Flügelparteien oder gar negative Mehrheiten drohten. Die speziellen rechtlichen Garantien zur Stabilisierung des Gleichgewichts der Staatsgewalten wurden durch den ganz allgemeinen Art. 25 WRV umgangen, leerlaufen lassen oder ignoriert. Das waren nicht bloß unbewusste Schritte auf einer abschüssigen Bahn, sondern auch politische Strategie des Verfassungswandels bzw. der Verfassungsreform. Sie wurde von der Staatsrechtswissenschaft teils vorbereitet, teils legitimiert. Und sie wurde zur politischen Praxis, seit die

53 Ausführlich Hoppe, Von der parlamentarischen Demokratie zum Präsidialstaat, 1998, S. 70 ff (Nachwe.).

54 Winkler, Weimar 1918-1933, 1993, S. 334 ff, 375 ff, 477 ff, 557 ff (hier auch der Terminus: „Auslieferung). Zu „Weimars Ende und Untergang“ Grimm, in: Dreier/Waldhoff aaO., S. 263 ff.

55 Zur Lehre von den Kampfregierungen Grimm, in: Winkler (Hg.), Die deutsche Staatskrise 1930 - 1933, 1992 , S. 155. 
Gegner der parlamentarischen Demokratie in immer mehr führende Ämter einrückten. Ihr Vabanquespiel konnte zwar die demkratischen Parteien disziplinieren, aber nur um den Preis einer Aushöhlung ihrer politischen Basis und einer Auslieferung der Republik an ihre Gegner. Das war ein Elitenversagen, was in neuerer Zeit als ein zentraler Grund des Scheiterns der Republik angeführt wird. Jedenfalls erfolgte die Kündigung des Verfassungskonsenses nicht nur durch die radikalen Parteien und ihre Wähler „von unten“, sondern auch durch Inhaber höchster Ämter „von oben“.56

Dabei war jenes Vabanquespiel Anfang der 30er Jahre nicht notwendig die Option für Hitler. Aber es hat die Republik destabilisiert, ohne eine handlungsfähige Alternative an ihre Stelle zu setzen. In diese Lücke konnte dann die stärkste verfassungswidrige Kraft, die Nationalsozialisten, einrücken. Im Sog ihres Erfolges auf der Ebene der Republik standen dann ihre Gewinne auf Länder- und Kommunalebene: Seltener vorher und als Experimentierfeld; häufiger als Nachvollzug der Fehleinschätzungen und falschen Weichenstellungen auf der Zentralebene. Das war damals auch unter den Aspekten von Verhältniswahl und parlamentarischer Regierungsform nicht zwingend. Wo es in der Republik präsidiale Gegengewichte oder Reservekompetenzen gegen die Volksvertretung nicht gab, war die parlamentarische Regierungsbildung alternativlos. In wichtigen Ländern gelang die Stabilisierung trotz Verhältniswahlrechts und Parteivielfalt. Parlamentarisches Regieren war auch damals nicht unmöglich. Von daher gewinnt die These an Plausibilität, wonach neben einer ständigen Überforderung der Republik und ihrer Regierung ${ }^{57}$ die Ausgestaltung und vor allem die Disfunktionalisierung ihres Organisationsrechts die Schwäche die gubernative Schwäche begünstigten.

\section{PLEBISZITÄRE REPUBLIK?}

Die im Verfassungstext detailfreudig ausziselierten Volksabstimmungen auf Antrag der obersten Reichsorgane fanden niemals statt, ihre Regelungen blieben totes Papier. Die in den Verfassungsberatungen schwach angeklungenen, später oft betonten und kritisierten Lehren vom Volk als Schiedsrichter zwischen den gewählten Organen 58 wurden nicht praktisch. Einzig relevant waren Plebiszite nach Volksbegehren. Sie unterlagen mehreren Prämissen: Zunächst der Begrenzung auf den Bereich der Gesetzgebung einschließlich verfassungsändernder Gesetze. Die Praxis verfuhr großzügig. Mindestens zwei Volksbegehren auf Reichsebene - „Panzerkreuzer" und „Freiheitsgesetz" - waren nur der Form nach Gesetze. In der Sache ging es um Einzelmaßnahmen der Regierung bei der Rüstungs-

$56 \mathrm{Zu}$ dieser Ursache des Niedergangs parlamentarischer Demokratien in der Zwischenkriegszeit Kershaw, Höllensturz, 2015, S. 178 ff, 263 ff. Zur Strategie Hindenburgs Pyta, Hindenburg, 2007, S. 479 ff, 489 ff, 555 ff u. pass. Dabei kommt es nicht auf die Frage an, ob dies eine Strategie seiner Person oder des von ihm installierten Systems und der in ihm agierenden Gegner der parlamentarischen Republik war.

57 Dazu Büttner, Weimar - Die überforderte Republik, 2008. Zur überforderten Verfassung Gusy, DSt 2016, 291.

58 Zu diesen Lehren und ihren Schwächen Gusy, Jura 1995, 226, 228. Zur Praxis Schiffers, Elemente direkter Demokratie im Weimarer Regierungssystem, 1971, S. 195 ff, 243 ff; Schwieger, Volksgesetzgebung in Deutschland, 2004, 25 ff. Zum Volksentscheid über die Auflösung des Preußischen Landtags (1931) Winkler, Der Weg in die Katastrophe, 2. A., 1990, S. 385 ff. 
politik bzw. die Unterzeichnung des Young-Plans. ${ }^{59}$ Sodann dem Art 73 Abs. 4 WRV: Haushaltsplan, Abgaben- und Besoldungsgesetze durften nur auf Antrag des Reichpräsidenten Gegenstand von Plebisziten werden. An diesen Hürden scheiterten auf Reichsebene 4 Volksbegehren. Die hohen Antrags- und Beteiligungsquoren verlangten schon für die Zulassung $10 \%$ der Stimmberechtigten, nur ausnahmsweise sollten $5 \%$ ausreichen (Art. 73 Abs. 2, 3 WRV). In der nachfolgenden Abstimmung genügte die einfache Mehrheit der Stimmen (§ 21 VolksentscheidG). Sollte ein Beschluss des Reichstags außer Kraft gesetzt werden, war ein Beteiligungsquorum von 50 \% vorgeschrieben (Art. 75 WRV). Dazu reichte der Parlamentsbeschluss aus, dem Volksbegehren nicht Folge zu leisten. So lag das hohe Quorum faktisch in der Hand des Parlaments, dem gegenüber die Korrekturfunktion ausgeübt werden sollte. Ein solcher Beschluss erging bei allen zugelassenen Volksbegehren.

Allein wirksam wurde die Korrektivfunktion des Volkes gegenüber den Parlamenten. Doch auch sie sollte die Ausnahme bleiben. Eindeutig fällt die Bilanz für die Reichsebene aus. Von den acht eingeleiteten Volksbegehren wurden drei nicht zugelassen, weil sie finanzwirksame Zielsetzungen aufwiesen, zwei weitere schon im Stadium der Unterschriftensammlung der Initiatoren nicht weitergeführt. Von den übrigen drei Begehren verfehlte eines das Quorum des Art. 73 Abs. 3 WRV: Gegen den Bau der Panzerkreuzer votierten nur 2,94 \% der Bürger. Die Begehren „Fürstenenteignung“ und „Freiheitsgesetz“, welche das 10 \%-Quorum erreichten, scheiterten im Volksentscheid an den Hürden des Art. 75 WRV. Solche Ergebnisse waren gewiss nicht geeignet, die Republik aus den Angeln zu heben. Auch das Gesamtbild in Republik und Ländern ist geeignet, diesen Eindruck zu verstärken: Zwischen 1919 und 1932 fanden sich etwa 60 Anläufe zu Volksbegehren, zumeist nach Landesrecht. Von ihnen wurde die knappe Hälfte zugelassen. In 12 Fällen fand daraufhin eine Abstimmung statt. „Erfolgreich“ war lediglich ein Fall: Auf Antrag von NSDAP und KPD wurde 1932 der Oldenburgische Landtag aufgelöst. Nach den notwendigen Neuwahlen regierte dort die NSDAP allein. Das ist keine Erfolgsgeschichte.

Nimmt man hingegen als Indikator einer Entfremdung zwischen (Gruppen von) Wählern und Gewählten nicht den Erfolg, sondern das Stattfinden eines Volksbegehrens, so zeigte sich: Notwendige Voraussetzung jeder Erfolgschance auch bei Volksbegehren war, dass Parteien die Initiativen unterstützten. Auch hier war der Parteienstaat demnach nicht der „geborene“ Gegner, sondern Erfolgsbedingung der politischen Wirksamkeit des Volkes. Die Plebiszite waren keine Äußerungsformen des Volkes ohne oder gegen die Parteien, sondern mit den und durch die Parteien. Direkte Demokratie und Parteienstaat waren auch damals keine Gegensätze, sondern ergänzten einander. Die meisten Initiativen fanden sich in den Jahren 1924-1926, kamen jedoch oft nicht über Vorbereitungshandlungen hinaus. Die relativ größte Häufung von Volksbegehren, die in Abstimmungen einmünden sollten, wurde für 1924 und 1931/32 festgestellt. Sie folgten der Krisenchronik der Republik und werden als Ausdruck des Protests in existentiellen wirtschaftlichen und politischen Krisen gewertet. Dafür sprechen auch andere Indikatoren. Initiiert wurden die Volksbegehren von rechten und linken Flügelparteien, auf welche etwa ein Viertel zurückging, und von Vertretern regionaler

59 Poetzsch-Heffter, JW 1929, 3364; s.a. Preuß, DJZ 1924, 649, 653 f; Schmitt, Volksentscheid und Volksbegehren, 1927, S. 45 ff. 
oder wirtschaftlicher Sonderinteressen des sich radikalisierenden Mittelstandes, also der Opfer der ökonomischen Katastrophen der 20er Jahre. Sie erreichten allerdings das Antragsquorum praktisch nie. Das gilt selbst für den viel diskutierten Sonderfall der Abstimmungskampagne gegen den für Deutschland eigentlich günstigen Young-Plan: Er war als Plebiszit über Reparationen geplant. Diese Frage stand gewiss nicht zur alleinigen Entscheidung der Deutschen. Unter den Initiatoren trafen sich erstmals bürgerliche Rechte und Völkische, also die spätere Harzburger Front, die Unterstützer Hitlers bei den Reichspräsidentenwahlen 1932 sowie der Koalitionsmehrheit vom Januar 1933. Dieser erste Anlauf zu dem Versuch, der jungen Republik die Sterbeglocke zu läuten, ${ }^{60}$ hat der im Niedergang befindlichen Republik schweren Schaden zugefügt. Angesichts dessen erscheint seine Unterstützung durch lediglich $14 \%$ der Wähler geradezu als Ausdruck staatspolitischer Reife des Volkes auch in schwerer Zeit. Viel spricht dafür, dass die Volksbegehren eher Krisenindikatoren als Krisenursachen waren. Ein wesentlich höheres Destruktionspotential sollte dagegen dem Appell an das Volk „von oben" durch Reichstagsauflösungen und Neuwahlen zukommen. Sie häuften sich ohne rechtliche Notwendigkeit seit 1930, also auf dem Höhepunkt der Wirtschafts- und Staatskrise.

In der Staatsrechtswissenschaft der Republik spielten Plebiszite nur eine periphere Rolle. ${ }^{61}$ Verfassungsloyale Autoren waren skeptisch gegenüber dem angeblichen „Volkswillen“. Sie waren auf der Suche nach gehaltvollen Demokratiekonzepten, welche das "Volk“ nicht bloß als ideale Zurechnungseinheit sah. Hier entstanden unter den Paradigmata von Freiheit, Gleichheit und Demokratie der Bürger Anläufe zu pluralistischen Konzepten. Danach sei „der“ Wille "des“ Volkes nicht einfach da und vorauszusetzen, sondern herstellungs- und feststellungsbedürftig. Pluralismus war danach nicht bloß ein empirisches Phänomen, sondern von den Grundrechten der WRV vorgegeben und der demokratischen Staatsform aufgegeben. Der Wille eines pluralistisch organisierten Volkes bedürfe der Formen und Verfahren durch Recht. Von daher gelangten sie zu einem institutionell geprägten Volks-, Staats- und Rechtsverständnis, welches einen Primat von Wahlen und Parlamenten als Mechanismen der Volkswillensbildung und -betätigung nahelegte. In der Republik wurden die Grundlagen gelegt für diejenigen Pluralismustheorien, welche seit 1945 in Deutschland als führendes Deutungsmuster zum Staat des GG galten. Für deren Anhänger war schon vor 1933 demokratisches Denken in der Republik zentral parlamentarisches Denken. Der auf demokratischen Wahlen fundierende, in öffentlicher Debatte diskutierte Parlamentswille sei der Volkswille und nicht dessen Entmündigung. Wahlen und Abstimmungen erschienen so als überlegene Formen der Äußerung des Volkswillens, gegenüber Plebisziten rechtlich mindestens gleichrangig. Vor diesem Hintergrund wurden Plebiszite als subsidiäre Korrektive gegenüber ausnahmsweisen Fehlent-

60 Kühne, Zeitschrift für Gesetzgebung 1990, 116, 120; s.a. Dreier, Merkur 2009, 1151, 1153: „Absage an derart populistische Projekte“.

61 Thoma, HDStR I, S. 192 (Ventil). Kelsen, Verhandlungen des 5. Deutschen Soziologentages, 1927, S. 37, 44; Anschütz, WRV (Anm. 21), S. 399 f (Nachw. zum Schutz des Parlaments). Zum gegenstandserzeugenden Demokratiekonzept Lepsius, in: Gusy (Hg.), Demokratisches Denken in der Weimarer Republik, 2000, S. 366, 397 ff. Zur Institutionenwahrnehmung in der Republik Möllers, in: Gusy ebd., S. 415. Späte Wendung zum Vorrang des Volkswillens bei Thoma, HDStR II, S. 114 ff, 166 (Nachw. zur Gegenauffassung). Überblick: Groh, Demokratische Staatsrechtslehrer in der Weimarer Republik, 2008, S. 300 ff (Nachw.). 
wicklungen in den Volksvertretungen angesehen. Das Regel-AusnahmeVerhältnis zwischen repräsentativer und unmittelbarer Demokratie konnte sowohl die Zulassung der Plebiszite wie aber auch deren Grenzen und hohen Hürden erklären. Sie sollten dem Schutz der prinzipiell parlamentarischen Volksund Staatswillensbildung dienen und wurden weniger als deren Grenzen, sondern als deren Effektivierung und Schutz gedeutet.

Aber auch dort, wo jene Ausgangspunkte nicht geteilt wurden, erlangten die plebiszitären Elemente der WRV damals wenig Aufmerksamkeit. Kritik etwa an Grenzen, strikten Verfahren oder hohen Quoren fand sich in Einzelfällen. Doch schlug der bereits genannte antiparlamentarische Affekt kaum in eine Präferenz für Volksentscheide nach der WRV um. Der „wahre“ Wille des „wahren“ Volkes werde durch Verfassungen nicht konstitutionalisiert, sondern liege ihm voraus. In diesem Sinne würden die Bürger erst durch den gemeinsamen Willen zum Volk als unmittelbar abstimmendes, präsentes und öffentlich handelndes Volk nach Art der Schweizer Landgemeinde oder der Kirchengemeinde im Gottesdienst. Auch wenn dieser Gesamtwille nicht notwendig der Wille Aller sei, so liege er den Wahlen und Abstimmungen voraus. Geheime Abstimmungen brächten jenen Gesamtwillen nicht zum Ausdruck, sondern „vernichteten“ ihn. ${ }^{62}$. Daraus folgte eine anti-institutionalistische Sichtweise, welche sich nicht allein gegen den Parlamentarismus, sondern auch gegen die plebiszitären Regeln der WRV richtete. Sie seien dem Wahlrecht angenähert, auf geheime Abstimmung und nicht auf Akklamation angelegt, also nahezu parlamentarisiert. Damit blieb ihr konstruktives Potential am ehesten auf die Verfassunggebung begrenzt. War die Verfassung in Kraft, erschien sie wegen ihrer Institutionalisierung und Formalisierung eher als Grenze der Demokratie und des Plebiszits. Wichtigstes Einzelergebnis war die These vom Vorrang der plebiszitären Legitimität vor der gesetzgebungsstaatlichen Legalität und damit das Postulat mindestens limitierter Abänderungsfestigkeit plebiszitärer Gesetze gegenüber parlamentarischen Modifikationen. So gering die rechtliche und politische Bedeutung der Plebiszite, so gering sollte auch der verfassungsrechtliche Ertrag der zeitgenössischen Diskussion um sie bleiben - sieht man von der Fundierung und der Kritik der Pluralismustheorien in Deutschland $\mathrm{ab}$.

\section{DAS FREIESTE VOLK DER ERDE“ - DIE GRUNDRECHTE}

Als der Präsident der Nationalversammlung Fehrenbach die Verabschiedung der neuen Verfassung mit diesen Worten feierte, 63 meinte er nicht deren Grundrechtsabschnitt oder deren Schutz durch eine Verfassungsgerichtsbarkeit allein. Er meinte vielmehr das Prinzip der Selbstbestimmung des Volkes durch die Demokratie. Sie war für ihn - neben der Gleichheit - die Staatsform der Freiheit und die Freiheit war Grundlage der Demokratie. ${ }^{64}$ Der Zweite Abschnitt war erst suk-

62 Schmitt, Volksentscheid und Volksbegehren, 1927, S. 33 f; 49 f; zurückhaltend Smend (Anm. 34), S. 119, 155: Politisches Volk hat sein Dasein „in erster Linie“ in staatlicher Wirklichkeit und Verfahren. Zu Verfassunggebung und Grenze Schmitt, Verfassungslehre, 1928, S. 86, 276 f. Zur Legitimität Schmitt (1932), in: Verfassungsrechtliche Aufsätze, 2. A., 1973, S. 263, 312 ff, unter Hinweis auf Jacobi, FS für das RG, 1929, S. 233. Zum Abbild-Denken dieser Richtung Lepsius, in: Gusy, Demokratisches Denken ebd., S. 376 ff.

63 Fehrenbach, in: Verh. des RT, Bd. 329, S. 2195.

$64 \mathrm{Zu}$ ihr Cancik, in: Dreier/Waldhoff aaO., S. 151. 
zessive den anfänglichen Plänen von Preuß hinzugefügt worden. Er war die eigenständigste und bleibende Leistung des Verfassungsausschusses, die im Plenum der Nationalversammlung umstritten blieb und die wohl schwerste Krise der Weimarer Verfassunggebung auslöste. Das eingangs angeführte Zitat illustriert die Grundrechtserwartungen in den Koalitionsparteien wohl zutreffend. Sie gingen zu Recht davon aus, dass Freiheit und Gleichheit sich nicht mit einem Federstrich anordnen ließen, sondern der Ausgestaltung und Verwirklichung durch Gesetze bedürften. Zu deren Umsetzung waren zahlreiche Zielbestimmungen, Gesetzgebungsaufträge und -vorbehalte statuiert. In ihnen ermächtigte das Volk als Verfassunggeber sich selbst zur Herstellung und Ausgestaltung der Grundrechte. Durch sie sollte also das freie Volk seine eigene Freiheit selbst bestimmen. Das war einerseits ambitioniert, andererseits voraussetzungsvoll, setzte es doch eine verfassungsloyale Gesetzgebung für die Zukunft voraus. Zugleich ging dieses Selbstverständnis einher mit einer gewissen normativen Schwäche des Grundrechtsabschnitts. Die ursprünglich vorgesehene Anordnung seiner unmittelbaren Geltung wurde wegen der Unabsehbarkeit ihrer möglichen Folgen zunächst abgeschwächt und sodann ganz gestrichen. ${ }^{65}$ Desungeachtet wurde ihre unmittelbare Geltung in der verfassungsloyalen Rechtswissenschaft angenommen.66 Doch konnten sie diese Wirkungen am ehesten entfalten, wenn sie als neuere Regelungen mit älteren Vorschriften kollidierten, welche durch die neuen Grundrechtsschrankenbestimmungen nicht gerechtfertigt werden konnten. $\mathrm{Ob}$ und wann dies der Fall war, blieb in nahezu jedem Einzelfall umstritten: Die Rechtsprechung nahm dies nur zögernd und zurückhaltend an. Daran änderte auch der damals heftige Streit um das Richterliche Prüfungsrecht wenig.

So sollten also rhetorische Stärke, inhaltliche Dichte und normative Schwäche zugleich den Zweiten Hauptteil der WRV prägen. ${ }^{67}$ Er enthielt ein ebenso komplexes wie zukunftsweisendes Programm: Die oben in der Einleitung genannten zukunftweisenden Verfassungsgehalte fanden sich ganz überwiegend im Zweiten Hauptteil. Lässt man einzelne sprachliche Eigenheiten der Zeit beiseite, so klingen dort zahlreiche Grundrechtsideen an, welche später als solche der zweiten oder dritten Generation bezeichnet wurden und bis in die jüngste Zeit Inhalte einzelner Verfassungen in anderen Staaten oder deutschen Bundesländern, aber auch der Europäischen Grundrechtecharta geworden sind. Gewiss waren dies keine unmittelbaren Folgen der Weimarer Garantien. Doch weisen diese so bis in die Gegenwart hinein und über sie hinaus.

Einen Ausgangspunkt bilden rechtsstaatliche Garantien, also zumeist klassische Abwehrrechte der Bürger gegen staatliche Übergriffe. Sie folgten teils den Vorbildern der Paulskirchenverfassung von 1849, teils denjenigen der deutschen Länderverfassungen. Das waren Ausprägungen bürgerlicher Errungenschaften der deutschen Verfassungsgeschichte. Neu sollte in diesem Kontext auch die Begründung des Willkürverbots durch Gleichheitsgarantien wirken. Und neu waren die explizite Anordnung des Gerichtsschutzes durch Verwaltungsgerichte und die Einführung einer Verfassungsgerichtsbarkeit, die allerdings damals nur be-

65 Dazu Kühne, Festschrift für Rudolf Wendt, 2015, S. 237.

66 Nachw. bei Dreier, in: ders./Waldhoff aaO., S. 175; zur Praxis Cancik ebd., S. 151; Gusy, ZNR 1993, 163.

$67 \mathrm{Zu}$ ihm nun ausführlich Cancik, Dreier, Stolleis in: Dreier/Waldhoff aaO., S. 151, $175,195$. Zu den übereinstimmenden Verfassungen Wiederin, in Dreier/Waldhoff ebd., S. 57 ff (Nachwe.). 
schränkt zugänglich war. Einstweilen sollten die vorhandenen Gerichte die Grundrechtssicherung übernehmen.68 Für die Zeitgenossen war dieser Grundrechtsschutz Ausprägung und Inhalt der Rechtsstaatlichkeit. $\mathrm{Zu}$ einer weitergehenden eigenständigen Rechtsstaatsdogmatik ist es damals nicht gekommen. Rechtsstaat war und blieb ein Staat mit Grundrechtsgarantien. In einem weiteren Sinne wurde er vereinzelt als Staatstyp qualifiziert: Bisweilen im Sinne von Verfassungsstaat; bisweilen als Staat des gleichen Rechts in Abwehr gegen das angeblich ungleiche Recht der Demokratie. ${ }^{69}$

Daneben enthielt die WRV umfassende sozialstaatliche Garantien. Weit davon entfernt, unerfüllbare Versprechungen aneinanderzureihen, enthielt sie differenzierte Garantien für die Zuweisung wirtschaftlicher Freiheiten an die dort tätigen Unternehmer und Arbeitnehmer; Schutz der Arbeitskraft und der geistigen Arbeit; den Schutz des Existenzminimums durch menschenwürdige Löhne und Arbeitsbedingungen und subsidiär staatliche Versicherungsleistungen gegen die zentralen Risiken des Lebens und öffentliche Sozialleistungen; den Vorrang der Eigenverantwortlichkeit vor der subsidiären staatlichen Zuständigkeit; Koalitionsfreiheit, wirtschaftliche Mitbestimmung sowie Sozialpflichtigkeit des Eigentums und der Wirtschaftstätigkeit in Abwägung mit einem umfassenden - ansatzweise auch im späteren Wortsinne ökologisch - verstandenen Gemeinwohl. In einem weiteren Sinne zählten dazu Rechte der Kinder auf Bildung und Erziehung, der Menschen auf Teilhabe an Infrastrukturen und Kultur. Das war sowohl Vorgabe wie aber auch Aufgabe eines gemeinwohlverträglichen Wirtschaftslebens; gestuft zwischen unmittelbar anwendbaren Grundrechten und Gesetzgebungsaufträgen. Auch hier sollte gelten: Zieht man einzelne sprachliche Zeiteinflüsse ab, so zeigen sich Grundzüge einer individualnützigen und sozial- wie umweltverträglichen Gestaltung von Arbeit, Wirtschaft und Politik, welche weit über die Republik hinaus Vorbildcharakter einnehmen konnte und bis in die Gegenwart hinein als Zielvorstellung anerkannt ist. ${ }^{70}$ Vor dem Hintergrund der nahezu permanenten wirtschaftlichen Misere der Zeit nahmen sich die Leistungen der Republik zur Umsetzung jener Vorgaben beachtlich aus. ${ }^{71}$

Weniger ausgeprägt, aber in Ansätzen vorhanden waren politische Teilhabe-, Mitwirkungs- und Kontrollrechte der Menschen. ${ }^{72}$ Die Selbstgestaltung der Gesellschaft durch Verbände und Organisationen; politisch ausübbare Grundrechtsgarantien (ältere Beschränkungen gegen politische Organisationen wurden ausdrücklich aufgehoben); staatsbürgerliche Gleichheit und ausdifferenzierte Wahl- und Abstimmungsrechte einschließlich ihrer Garantie für die Frauen; Subsidiaritätselemente durch Selbstverwaltung und öffentliche Körperschaften; gleicher Zugang zum öffentlichen Dienst; Gleichstellung besonderer vulnerabler Gruppen; aber auch einzelne politische Grundpflichten: Hier zeigten sich Grund-

68 Diese Intention wurde durch den damaligen Streit über das richterliche Prüfungsrecht partiell überdeckt. Zu diesem Wendenburg, Die Debatte um die Verfassungsgerichtsbarkeit und der Methodenstreit in der Staatsrechtslehre der Weimarer Republik, 1984.

69 Überblick bei Gusy, in: Jouanjan u.a. (Hg.), Figures de L’État de droit, 2001, S. 331.

70 Eichenhofer, Quaderni Fiorentini 2017, 199; Stolleis, in: Dreier/Waldhoff aaO., S. 195.

71 Näher Berg, in: Deutsche Verwaltungsgeschichte IV aaO.

72 Deren Erschließung ist partiell noch Desiderat; s. einstweilen Gusy, 100 Jahre WRV, 2018, S. $263 \mathrm{ff}$. 
züge einer politischen Kultur, die auf Aktvierung der Gesellschaft, Demokratisierung des Staates und ansatzweise der Wirtschaft; Öffnung der öffentlichen Gewalt für das Volk sowie Mitwirkungs- und Kontrollrechte der Bürgerinnen und Bürger angelegt war. Dazu zählte nicht zuletzt die große Zahl von Gesetzgebungsaufträgen und Gesetzesvorbehalten, welche die Gestaltung des Gemeinwesens durch die demokratische Legislative vorgab und vorschrieb. Das waren damals eindeutige Positionen gegen die tradierte Monarchie und ihre Staatsgewalt von oben nach unten. Und diese Errungenschaften waren damals alles andere als selbstverständlich, ausbaufähig und auf gesetzlichen Ausbau angelegt. Und dieser war der Legislative als demokratische gewähltes Organ im Staat als Selbstorganisation der Gesellschaft überantwortet. Emphatisch formuliert sollte sich das Volk seine Rechte selbst nehmen und gestalten können.

Der Zweite Hauptteil war weit davon entfernt, bloß die tradierte Trennung von Staat und Gesellschaft und den Schutz der letzteren durch Abwehrrechte vorzusehen. Vielleicht war es genau dies, war eine distanzierte Aufnahme seiner Garantien in Teilen der Staatsrechtswissenschaft motivierte. Sie mobilisierte das tradierte Denken der Disziplin gegen die neuen Garantien der Verfassung. Dabei war sich der Verfassungsausschuss der Programmatik seiner Vorgaben ebenso bewusst wie der Mühen ihrer Verwirklichung: Dass hier auf den Gesetzgeber zahlreiche und große Aufgaben zukommen würden, zeigten schon die zahlreichen Gesetzgebungsaufträge. Den Weimarer Abgeordneten war nur zu bewusst, dass der Weg zu realer Freiheit, angemessenem Lebensstandard und effektiver Teilhabe am gesellschaftlichen Leben für Alle ein weiter Weg sein würde. Insoweit waren normative Schwäche, Umsetzungs-und Ausgestaltungsnotwendigkeiten durch die Legislative, Abwägungsoffenheit der Rechte untereinander und mit öffentlichen Belangen nicht bloß Schwächen, sondern Stärken ihres grundrechtlichen Programms. Dieses war eben nicht nur auf Freiheit vom Staat, sondern eben auch auf Freiheit im Staat und durch den Staat angelegt. Hier sollte sich erneut der Kontext von Freiheit und Demokratie zeigen: Demokratie ist grundrechtsaffin. Sie basiert auf Grundrechten und ist besonders dazu geeignet, Freiheit der Menschen zum Auftrag, Inhalt und Gegenstand der öffentlichen Gewalt zu nehmen. Und Freiheit wurde auch politisch gedacht und damit demokratieaffin. Im Jahr 1919 war das demokratische Grundrechtsdenken sehr ausgeprägt: Aber was damals als widersprüchlich und kompromisshaft kritisiert worden ist, würde in der Gegenwart nach den Grundsätzen der praktischen Konkordanz und des schonendsten gegenseitigen Ausgleichs abgearbeitet werden. Eine gewisse Schwäche jenes Konzepts mag man aus der Retrospektive in einer gewissen Unterkomplexität sehen: Namentlich der Schutz von Minderheitenrechten in der auf Mehrheitsherrschaft angelegten Demokratie wurde seinerzeit angemahnt. Aber selbst hier war die WRV sensibel: Sie schrieb eine Verwaltungsgerichtsbarkeit vor und errichtete mit dem Staatsgerichtshof eine der ersten Verfassungsgerichtsbarkeiten der Welt. Zwar kannte dieser damals noch keine Verfassungsbeschwerde. Ihre Einführung war aber auch nicht ausgeschlossen. Und eine solche Rechtsschutzmöglichkeit kannte selbst das Grundgesetz im Jahre 1949 bekanntlich auch noch nicht. 


\section{NEUTRALITÄT BIS ZUM SELBSTMORD?- DAS NATIONALSOZIALISTI- SCHE REGIME ALS LETZTE REGIERUNG DER REPUBLIK?}

Die These von der legalen Machtübergabe an die Nationalsozialisten behauptete die Vereinbarkeit des Ermächtigungsgesetzes mit der WRV. Nach ihr war die WRV eine Verfassung, welche den Nationalsozialismus nicht verhindert, sondern legalisiert, wenn nicht gar ermöglicht habe. Hier trifft sich die Legalitätsthese mit der Neutralitätsthese. Die Weimarer Verfassung sei staatsformneutral gewesen. Auf der Grundlage des Art. 76 WRV sei nicht bloß die Abänderung, sondern auch die Abschaffung von Republik, Demokratie und Grundrechten möglich gewesen. Aus der Sicht mancher Betrachter schien sie noch weitergehend sogar neutral bis zum Selbstmord; genauer: bis zur Selbstaufhebung. Es gibt in der Rechtswissenschaft wohl kein Argument, welches schärfer als die Legalitätsthese akzentuiert, dass nicht allein Art. 76 WRV, sondern die Weimarer Verfassung insgesamt die falsche Verfassung einer falsch verstandenen Republik zur falschen Zeit gewesen sei. ${ }^{73}$

Ob Verfassungsänderungen in der Republik unbegrenzt zulässig waren oder auf rechtliche Schranken trafen, war damals sehr umstritten. Wer solche Grenzen bejahte, konnte oder wollte sie jedoch nicht immer konkretisieren. Ob etwa das parlamentarische Regierungssystem unantastbar sei, war für sie keineswegs sicher. Andere sahen für den demokratischen Prozess keine materiell-rechtlichen Schranken. Doch forderten sie, dass die Änderung durch eine demokratische Entscheidung getroffen werde. Wie eine solche getroffen werden könne, richtete sich für sie nach der WRV selbst. Insoweit war auch aus dieser Sicht weder die WRV selbst noch der Art. 76 WRV neutral. Im Gegenteil: Art. 1 WRV statuierte die demokratische Republik; und über deren Einschränkung, Abänderung oder Abschaffung durfte gem. Art. 76 WRV allein nach den Regeln dieser Republik entschieden werden. Darin lag keine Distanzierung der Verfassung von der selbst konstituierten Staatsform, sondern eine eindeutige Parteinahme: In möglichen Auseinandersetzungen um die Demokratie verwies die Weimarer Verfassung auf die Regeln eben dieser Demokratie. Sie selbst und insbesondere die Bestimmungen über die Verfassungsänderung standen also fest auf dem Boden der eigenen Grundlagen. Die Möglichkeit einer „scheinbar legalisierten Methode des Staatsstreichs“ (Thoma) blieb immer noch an die Regelungen der WRV einschließlich ihres Demokratieprinzips gebunden. Dieses war auch in der Republik kein bloßer Kampf um die Macht, sondern ein politischer Prozess nach den Regeln der Verfassung und der verfassungskonformen Gesetze. Sie forderten nach Auffassung verfassungsloyaler Autoren zentral die "geistige“ Auseinandersetzung mit den in der WRV zugelassenen politischen Aktivitäten in Medien, Versammlungen, Parteien und sonstigen Vereinen. ${ }^{74}$ Andere Instrumente im politischen Kampf waren danach nicht allein von der Normalität demokratischen Entscheidens, sondern

73 Zur Legalitätsthese in der Republik Schmitt (1932), in: Aufsätze (Anm. 40), S. 262; nach 1945 ders. (1950), ebd. S. 440. Zur Neutralitätsthese ders., Der Reichspräsident als Hüter der Verfassung, 1931, S. 132 ff. Zur Weimarer Legalitätskritik Schmitts Hofmann, Legitimität contra Legalität, 4. A., 2002. Scheinbar für Freiheit zur Selbstaufhebung und zum demokratischen Verfahren damals Thoma, HDStR I, S. 185, 193. Zum Folgenden Kelsen, Staatsform und Weltanschauung, 1933, S. 13 ff; Radbruch, Die Justiz, 1929, 125 ff; ders., (1946), in: ders., Gesamtausgabe, Band 3, 1990, S. 88 f. Nachw. bei Gusy in Neuhaus (Hg.), Verfassungsänderungen, 2012, S. 159, S. $159,171 \mathrm{ff}$.

${ }^{74}$ Zur Doppelstrategie der NSDAP Blasius, Weimars Ende, 2006 (Nachw.). 
auch von der demokratischen Verfassungsänderung ausgeschlossen. Sie waren eben nicht legal, sondern illegal - schon in der Republik. Bei genauem Hinsehen zeigt sich: Verfassungsloyale Autoren haben das verfassungsmäßige Recht der Republik, sich gegen die Extremisten von links und rechts $\mathrm{zu}$ wehren, nicht ernsthaft in Frage gestellt. Im Gegenteil: Bei ihnen fanden sich demokratietheoretische und verfassungsjuristische Ansätze zu einer Lehre von der streitbaren Demokratie. ${ }^{75}$ Sie wurde damals noch nicht so genannt, doch wurden solche Ansätze nicht erst im Exil „erfunden“. Die Formel ist jünger, die Sache älter - eine Einsicht nicht erst aus der Republik, sondern im Ansatz schon in der Republik. Sie stand schon damals gegen die Neutralitätsthese, die sich erst ex post zur Beschreibung der WRV durchgesetzt hat.

Wie aber sah nach diesen Maßstäben die Machtübernahme der Nationalsozialisten aus? War das NS-Regime das „legale“ im Sinne der demokratischen Republik? Die der NSDAP für diese Zeit attestierte Doppelstrategie verknüpfte den gewaltsamen Straßenkampf mit parlamentarischer Destruktion. Wenn von letzteren jedenfalls einzelne verfassungsgemäß gewesen sein sollten, so galt das von ersteren auch nach damals einhellig vertretenem Rechtsverständnis nicht. Illegal waren auch damals Mord, Totschlag, Körperverletzung, Landfriedensbruch und Nötigung - auch als Mittel zum politischen Zweck. Es gab auch in der WRV kein uneinschränkbares Recht auf eine „nationale Revolution“. Die Legalitätsthese blendet einen zentralen Zweig des Kampfes der NSDAP gegen die Republik völlig aus. Dies entsprach den vielfältigen Windungen des Legalitätskonzepts der NSDAP. Seit ihrem Erstarken im Reichstag und der abnehmenden Furcht vor einem Parteiverbot hielt sie propagandistisch für „legal“, was der Bewegung nutzte; was also zur Verfolgung ihrer Zwecke legitim erschien - bis hin zum Mord. Hier fielen dann Legitimitätsideen und vorgebliche Legalitätsbehauptungen ineinander. Der Weg der NSDAP vom Münchener Landfriedensbruch bis zum PotempaMord war schon vor 1933 kein „legaler“ gewesen, wenn diesem Wort noch irgendeine Bedeutung zukommen soll. Dementsprechend hatten die regelmäßigen Legalitätsbehauptungen der NSDAP schon in der Republik ausschließlich taktischpropagandistische Funktion eingenommen, welche sich im Laufe der Jahre wandelte. Und was den Ereignissen vor 1933 vorausging, verschärfte sich unter Hitlers Kanzlerschaft: Auch wenn die eine oder andere Formalie „legal“ erschien, so häuften sich die Rechts- und Verfassungsbrüche. Nur aus einem Blickwinkel gegensatzaufhebender Begriffsbildung hätte auch der Straßenkampf „legal“ sein können. Aber ein solches Konzept war damals außerhalb der politischen Parteigänger der Flügelparteien von niemandem anerkannt.

Bekanntlich war ein zentraler Grund für das Scheitern der Republik die Kündigung des Verfassungskonsenses „von oben“. Und wie legal war dieses Vorgehen? "Legal“ war noch der formale Akt der Ernennung Hitlers durch den Reichspräsidenten gewesen - die unterbliebene Konsultation des Reichstags entsprach dem damals schon erreichten Stand der Auflösung der WRV. Fortan setzte sich die Doppelstrategie der Nationalsozialisten fort: ${ }^{76}$ An die Stelle der bürger-

75 Einzelheiten bei Gusy, 100 Jahre Weimarer Verfassung, 2018, S. 319 ff.

76 Zum frühen Straßenterror der NS-Machthaber näher Grube/Richter, Alltag im Dritten Reich, 1982, S. 25 ff; Becker/Becker (Hg.), Hitlers Machtergreifung 1933, 3. A., 1993, S. 31 ff; psychologisch $v$. Hentig, Terror, 1970. Zur Psychologie der Machtergreifung, 1970; literarisch Liepmann, Das Vaterland, 1934/1966. Zur SA als Hilfspolizei Erlass des Preußischen Innenministers (Göring) v. 22.2. 1933, II C I 59 Nr. 40/33. ReichstagsbrandVO v. 28.2. 1933, RGB1 I 83. 
kriegsähnlichen Unruhen trat nun der staatlich teils organisierte, teils gebilligte Terror gegen tatsächliche oder vermeintliche Gegner. $\mathrm{Zu}$ seiner Rechtfertigung wurde keineswegs die Weimarer Verfassung bemüht. Eine notdürftige Kaschierung begründete die Notverordnung zum Schutz von Volk und Staat v. 28.2.1933, welche die meisten formellen und materiellen Grenzen des Art. 48 Abs. 2 WRV hinter sich ließ. Die Reichstagswahlen vom März 1933 waren unter den Bedingungen von Terror, Einschüchterung, Versammlungs- und Veranstaltungsverboten von den Grundsätzen der Art. 22 WRV weit entfernt. Kulminationspunkt der Verfassungsauflösung war das Ermächtigungsgesetz vom 24.3.1933. Die Abwesenheit der 81 verhafteten oder verfolgten KPD-Abgeordneten war nicht allein ein Verstoß gegen deren Immunität und damit ihre individuellen Abgeordnetenrechte. Art. 37 WRV schützte nach damaligem Verständnis auch die Volksvertretung insgesamt gegen Einschüchterung und Beeinträchtigung des freien Mandats, und zwar auch gegen Eingriffe in das Mandat durch Maßnahmen gegen andere Abgeordnete. ${ }^{77}$ Insoweit handelten nicht nur die verhafteten oder geflohenen Mitglieder des Reichstags, sondern auch die abstimmenden Abgeordneten nicht mehr frei. Noch verstärkt wurde dieser Effekt durch die einschüchternden Begleitumstände der Reichstagssitzung. Die Behauptung, wonach das Ermächtigungsgesetz die erforderliche Mehrheit auch erlangt hätte, wenn den ablehnenden Stimmen der 94 SPD-Abgeordneten diejenigen der KPD-Fraktion hinzugerechnet würden, ändert an jenen rechtlichen Mängeln nichts. Das waren nicht „bloß“ formelle Mängel im Verfahren, sondern Verletzungen von Grundprinzipien der demokratischen Republik. Fehlerhaft war auch die Abstimmung im Reichsrat, in welchem entgegen dem Preußenschlag-Urteil des StGH auch Stimmen abgegeben wurden, welche von Reichskommissaren anstelle der abgesetzten Länderregierungen instruiert waren. Ob der Inhalt des Ermächtigungsgesetzes mit der WRV vereinbar war, ist angesichts des damals stark im Fluss befindlichen Meinungsstandes zu den Grenzen der Verfassungsänderung schwer zu beantworten. Gewiss ist: Das Gesetz ging weit über frühere Ermächtigungen hinaus und reduzierte die Kontrollrechte der ohnehin nicht mehr freien Volksvertretung auf ein Minimum. Jedenfalls auf Lehren, welche die frühen Ermächtigungsgesetze der Republik für zulässig erklärt hatten, konnte die Verfassungsmäßigkeit des Gesetzes von 1933 nicht gestützt werden.

Das Ermächtigungsgesetz war also formell nicht „legal“ und materiell kaum legitimierbar im Sinne der Weimarer Verfassung. Doch wurde die Verfassung auch nicht (erst) vom Ermächtigungsgesetz zerstört. Dass dieses unter den angedeuteten Vorgaben überhaupt so beschlossen werden konnte, wie es beschlossen worden ist, zeigt, wie sehr die WRV schon im März 1933 zerstört war. ${ }^{78}$ Die nationalsozialistische Herrschaft war nicht Ausdruck demokratischer Legalität im Rahmen der WRV, sondern Folge ihres tiefsten Niedergangs. Und sie fand nicht im Rahmen der WRV, sondern teils im Zuge ihrer Beseitigung und teils nach ihrer

77 Anschütz, WRV (Anm. 21), S. 232: nicht Privileg des Abgeordneten, sondern Privileg des Parlaments; Graf zu Dohna, HDStR I, S. 445 f: "nicht Vorrecht des einzelnen Abgeordneten, sondern Schutzrecht des Hauses“. Zu den historischen Fakten Morsey, Das Ermächtigungsgesetz vom 24. März 1933, 2. A., 1992, S. 129 ff; Heuss, Die Machtergreifung und das Ermächtigungsgesetz, 1967; zu ihrer rechtlichen Würdigung Dreier, Merkur 2009, 1151, 1154 ff. Zur Ermächtigungsgesetzgebung in der Republik historisch Gusy, Zeitschrift für Neuere Rechtsgeschichte 2017, 246.

78 So die zusammenfassende These von Büttner (Anm. 3), S. 498 ff. Vertiefend Dreier (Hg.), Macht und Ohnmacht des Grundgesetzes, 2009. 
Beseitigung statt. Die WRV eröffnete keine (unbegrenzte) Freiheit zur Selbstaufhebung. Die Legalitätsthese stößt inzwischen weithin auf Ablehnung. ${ }^{79}$

\section{RÜCKBLICK: VON WEIMAR ZU „WEIMAR“ UND WIEDER ZURÜCK}

Nach 1945 wurden die Rückblicke auf die gescheiterte Republik und ihre Verfassung geprägt von den Fragestellungen und Antwortsuchen der zurückschauenden Autoren. Das Gefühl des „Nie-Wieder“ jedenfalls gegenüber der Hitler-Barbarei, die Suche nach Ursachen und nach Verantwortlichen überlagerte die meisten Diskussionen. Da demokratische Traditionen seit 1933 in Deutschland abgebrochen waren, wurden Erklärungen von Zeitzeugen geprägt, die damals selbst zu den Kritikern gezählt hatten. Sie sahen Ursachen und Verantwortung am ehesten dort, wo sie sie stets gesehen hatten. Dabei konnten sie an ihre frühere Verfassungskritik anknüpfen. Das Ende der WRV galt ihnen als Bestätigung ihrer früheren Auffassungen. So setzte sich jedenfalls diese Richtung aus dem Richtungsstreit nach 1945 fort, nämlich die Diagnose von den Mängeln und der Fehlerhaftigkeit der WRV. Die Verfassung - und nicht etwa der Widerstand gegen sie - sei ursächlich für den eigenen Untergang. Die Verantwortung konnte damit abgewälzt werde auf eine Institution, die sich nicht mehr wehren konnte: Die untergegangene WRV. Die langen Schatten der untergegangenen Republik ${ }^{80}$ legten sich auch auf deren rechtliche Grundordnung. An deren Stelle traten die aus ihr zu ziehenden Lehren. Weimar wurde zu „Weimar“.

Das war nicht mehr die Weimarer Verfassung, die von der Nationalversammlung verabschiedet und im Reichsgesetzblatt veröffentlicht worden war. Es war das Zerrbild, das im Zuge des Kampfes um die Republik aus ihr gemacht worden war. Und dieses Zerrbild sollte lange Zeit hindurch das Bild des Verfassungswerks überdauern und verdunkeln. In der NS-Zeit wurde sie keineswegs als die „neutrale“ Grundordnung gefeiert, welche die „legale Machtergreifung“ überhaupt erst möglich gemacht hatte. Sie wurde weiterhin perhorresziert, die Legalitätsthese verschwand rasch. Alsbald stützte sich die These von der Legitimität der nationalen Revolution und der Überwindung von Weimar weitgehend auf die angeblichen Mängel der Republik und ihrer „Systems“.

Nach 1945 hätte in der Bundesrepublik eine Revision jenes Geschichtsbildes einsetzen können. Dazu gab es eine Reihe von Ansätzen, weniger in der Theorie als in der Praxis. Die frühen Verfassungen einiger Bundesländer - etwa: Bayern, Hessen, Rheinland-Pfalz - wiesen Anleihen bei Aufbau und Text der WRV auf. Bis zur Schaffung des Grundgesetzes sollte sich dieses Bild ändern. Auch wenn im Parlamentarischen Rat vereinzelt betont wurde, die Weimarer Verfassung sei besser gewesen als ihr Ruf, so sollte sich doch die Sichtweise vom Grundgesetz als Kontrastfolie zu seiner Vorgängerin durchsetzen. Es galt lange Zeit hindurch als zentrale „Lehre“ aus dem Untergang der ersten deutschen Demokratie. Nun wurde das "Nie wieder" auch auf die Republik als Inkubationszeit von NS-Ideologie und NS-Herrschaft ausgeweitet. Ein neues Grundgesetz mit Aussicht auf Erfolg müsse anders sein als die gescheiterte Vorgängerin. Daher sei das Grundgesetz auf dem richtigen Wege, wo und weil es andere Inhalte aufweise als „Weimar“;

79 Ablehnend etwa Stolleis, Geschichte der Öffentlichen Rechts in Deutschland, Band 3, 1999, S. 246 f, 317 ff; Dreier, Merkur 2009, 1154 f; Gusy, WRV, S. 151 f, 417 ff, 459 ff; ders., in: Neuhaus (Anm. 41), S. 159, 179 ff (alle mit Nachw.).

80 Mommsen, in: Bracher/Funke/Jacobsen, Die Weimarer Republik, 3. A., 1998, S. 552; Gusy (Hg.), Weimars lange Schatten, 2003; Ullrich, Der Weimar-Komplex, 2009. 
und umgekehrt sei „Weimar“ auf dem falschen Weg gewesen, wo und weil es anders gelautet habe als die neue Grundordnung. Solche Vergangenheitsbewältigung wurde zur self-fulfilling-prophecy, seit Bundesrepublik und Grundgesetz sich als Erfolgsmodell erwiesen. Die „Lehren“ aus der Vergangenheit schienen dadurch bestätigt. Inzwischen sind die Thesen vom Gegenentwurf partiell überholt, partiell relativiert. Der Erfolg der Bundesrepublik hing jedenfalls nicht von den Unterschieden des GG gegenüber der WRV ab. Dass das Grundgesetz eine gelungene Verfassung ist, besagt noch nichts darüber, dass sein Weimarer Vorläufer und Gegenstück eine misslungene gewesen wäre.

In der jüngeren Verfassungsgeschichtsschreibung haben sich auch die Vergleichsmaßstäbe verändert. Verfassungsdokumente der späten 40er Jahre erschienen nicht nur in Deutschland oft minimalistisch. Hier wurden etwa politische und soziale Garantien zumeist ausgespart. Damit erschienen solche Dokumente schon äußerlich als Absagen an ältere Vorbilder nach Art der Weimarer Verfassung. Doch hat sich die Rechtskultur seitdem gewandelt. Jüngere Verfassungsdokumente in anderen Staaten und in Europa enthalten wie selbstverständlich auch soziale und politische Garantien, Volksabstimmungen und eine selbstbewusste, nicht bloß „streitbare“ Demokratie. ${ }^{81}$ Darin liegt gewiss primär eine Abweichung von den Traditionen der 40er Jahre. Zwar lässt sich eine Orientierung an der Weimarer Verfassung nur selten positiv nachweisen. ${ }^{82}$ Aber auch hier war die Weimarer Verfassung schon da - wie bei zahlreichen anderen Errungenschaften der jüngeren Verfassungsentwicklung auch. Doch waren ihre Qualitäten lange Zeit überlagert von ihrem allzu schlechten Image.

\section{DIE WRV ALS MEILENSTEIN DER DEUTSCHEN UND EUROPÄISCHEN VERFASSUNGSGESCHICHTE}

Dass eine überforderte Republik eine überforderte Verfassung hatte, liegt nahe. Im Nachhinein waren die langen Schatten der untergegangenen Republik so umstritten wie wenig anderes in der deutschen Verfassungsgeschichte. ${ }^{83}$ Für die NS-Zeit ist beobachtet worden, dass nahezu niemand im Widerstand gegen Hitler für eine erneuerte Republik nach Weimarer Muster gekämpft habe: Zu belastet und delegitimiert sei diese nach ihrem lautlosen Verschwinden gewesen. Ganz entgegengesetzt findet sich die Auffassung, wonach die Einführung einer funktionsfähigen Demokratie durch ausländische Truppen nach einem Krieg in der Welt nirgends gelungen sei - außer in Deutschland nach 1945. Dafür seien aktivierbare, lebens- und handlungsfähige demokratische Strukturen und Einstellungen ursächlich gewesen, welche man nur hier vorgefunden habe. Und deren letztes und wichtiges Betätigungsfeld war die Republik von Weimar mit ihrer Verfassung gewesen. Auch wenn berücksichtigt werden muss, dass beide Position unterschiedliche Zeitpunkte der Retrospektive betreffen, bleibt doch festzuhalten: Offenbar hatte auch die Rückschau auf die WRV ihre „Zeit der Extreme“.

81 Einschränkend zuletzt BVerfGE 144, 20. Historisierende Einordnung bei Ipsen, Recht und Politik 2017, 3.

82 S. dazu Wiederin, in: Dreier/Waldhoff aaO., S. 45, $57 \mathrm{ff}$.

83 Erste Position entwickelt bei Mommsen, in: Bracher/Funke/Jacobsen (Hg.), Die Weimarer Republik 1918-1933, 3. A., 1998, S. 552 ff, 561 ff. Zweite Position nach Ullrich (Anm. 47), S. 108 ff. $\mathrm{Zu}$ Möglichkeiten und Grenzen des Lernens aus der Verfassungsgeschichte Gusy, in: Stolleis, Verfassungs(ge)schichten, 2017, S. 65. 
Die Weimarer Verfassung war das Beste, was die freiheitlichen und demokratischen Verfassungstraditionen in Deutschland nach dem 1. Weltkrieg zu bieten hatten. Mehr an Verfassunggebung des Volkes hat es hier zu keinem Zeitpunkt gegeben. Das galt nicht allein für den bloßen Text. Wichtige Interpreten entdeckten schon damals ihre Vorzüge. Sie entwickelten Anläufe zu ambitionierten Rechts- und Sozialstaatslehren. Und sie entdeckten Grundlagen von Pluralismustheorien und streitbarer Demokratie, zur verfassunggebenden Gewalt des Volkes und der Einsicht, dass eine Verfassung nach Art der WRV des Engagements der verfassungsloyalen Bürger, Parteien und Interpreten bedarf. Dies alles hat sich nicht erst in der Bundesrepublik, sondern schon in der Republik entwickelt. Gewiss: Manches blieb damals bisweilen tentativ, entwicklungsbedürftig und vereinzelt spekulativ. Zu viel war neu zu denken, und zu kurz war die Dauer der Republik. Daran konnte nach 1933 angeknüpft werden - zunächst im Exil, sodann in der Bundesrepublik. Wir konnten und können aus Weimar mehr und anderes lernen als den staatsrechtlichen „Richtungsstreit" mit seiner Verfassungskritik. Es gab auch damals demokratisches Denken und eine verfassungsloyale Staatsrechtswissenschaft. Solche Bilder stellen diejenigen vom Beginn dieser Darstellung bisweilen geradezu auf den Kopf. ${ }^{84}$ Das bedeutet keineswegs, in Zukunft einfach die früheren negativen durch neue positive Vorstellungen zu ersetzen. Die Verfassung der ersten deutschen Republik war weder ein Mängelwesen noch aber auch gänzlich mängelfrei. ${ }^{85}$ Die Abkehr von den alten Bildern mag eine differenzierte, angemessen komplexe Betrachtung der Verfassung ermöglichen, die nicht nur alte Fragen durch neue ersetzt, sondern auch einzelne Antworten sucht und vielleicht findet.

Die Republik ging unter, als der ihr zugrunde liegende Verfassungskonsens aufgekündigt wurde. Damit bestätigt sich die Einsicht, dass freiheitliche säkulare Staaten von Voraussetzungen leben, die sie selbst nicht garantieren können ${ }^{86}-$ wie übrigens wohl alle anderen Verfassungen auch! Diese Voraussetzungen und ihre Gefährdungen stehen nicht ein für allemal fest, sie wandeln sich insbesondere unter den Bedingungen rechtlich garantierter Freiheit und Demokratie. Diese Einsicht begrenzt zugleich die Möglichkeiten, aus der Verfassungsgeschichte zu lernen. Rückblickend können wir Republik und ihre Verfassung, ihre Chancen und Risiken, ihre Stärken und ihre Mängel in die Tradition der westlichen Verfassungsstaaten einordnen. Aber jede Verfassung hat ihre Bewährungsprobe in der Gegenwart und in der Zukunft, nie in der Vergangenheit. Daher lassen sich die Anforderungen an die Bundesrepublik und ihre Verfassung nicht (bloß) durch Vermeidung der Fehler aus der ersten deutschen Republik bewältigen. Maßgeblich für den Erfolg der Bundesrepublik und des Grundgesetzes ist die Fähigkeit zur Meisterung der Anforderungen unserer Zeit. Die Geschichte wiederholt sich nicht - wohl aber liefert sie Erfahrungs- und Anschauungsmaterial zur Erhöhung des Diskussionsniveaus. Dieses Material richtig zu nutzen setzt eine möglichst große Annäherung nicht nur an die Gegenwart, sondern auch an die Vergangenheit voraus - nicht nur die Mängel und Grenzen, sondern auch die Vorzüge und Qualitäten der Weimarer Verfassung. Sie war mit allen Stärken und Schwächen

\footnotetext{
84 S. O. II.

85 Hinweise bei Stolleis, in: Dreier/Waldhoff aaO., S. 200.

86 Böckenförde (1967), in: ders., Recht, Staat, Freiheit, 1991, S. 92, 112.
} 
ein Meilenstein der deutschen und der europäischen Verfassungstradition - eine gute Verfassung in schlechter Zeit.

Fecha de envio / Submission date: 26/04/2019

Fecha de aceptación / Acceptance date: 8/05/2019 